\title{
Geochemistry of Soils and Streams on Surfaces of Varying Ages in Arctic Alaska
}

Authors: Keller, Katy, Blum, Joel D., and Kling, George W.

Source: Arctic, Antarctic, and Alpine Research, 39(1) : 84-98

Published By: Institute of Arctic and Alpine Research (INSTAAR), University of Colorado

URL: https://doi.org/10.1657/1523-

0430(2007)39[84:GOSASO]2.0.CO;2

BioOne Complete (complete.BioOne.org) is a full-text database of 200 subscribed and open-access titles in the biological, ecological, and environmental sciences published by nonprofit societies, associations, museums, institutions, and presses.

Your use of this PDF, the BioOne Complete website, and all posted and associated content indicates your acceptance of BioOne's Terms of Use, available at www.bioone.org/terms-of-use.

Usage of BioOne Complete content is strictly limited to personal, educational, and non - commercial use. Commercial inquiries or rights and permissions requests should be directed to the individual publisher as copyright holder.

BioOne sees sustainable scholarly publishing as an inherently collaborative enterprise connecting authors, nonprofit publishers, academic institutions, research libraries, and research funders in the common goal of maximizing access to critical research. 


\section{Geochemistry of Soils and Streams on Surfaces of Varying Ages in Arctic Alaska}

\author{
Katy Keller*t \\ Joel D. Blum* and \\ George W. Kling $\dagger$ \\ *Department of Geological Sciences, \\ University of Michigan, 1100 North \\ University Avenue, Ann Arbor, \\ Michigan 48109, U.S.A. \\ $\dagger$ Department of Ecology and \\ Evolutionary Biology, University of \\ Michigan, 830 North University \\ Avenue, Ann Arbor, Michigan 48109, \\ U.S.A. \\ †kateak@umich.edu
}

\begin{abstract}
Climate change may bring about geochemical changes in arctic regions as a result of increasing thaw depth. In order to better understand current watershed geochemistry and mineral weathering and provide a basis for predicting the geochemical effects of active-layer thickness increase, we examined elemental chemistry and ${ }^{87} \mathrm{Sr} /{ }^{86} \mathrm{Sr}$ of streams and sequential and total digests of soils, permafrost, and soil parent materials from seven glacial deposit surfaces of varying geomorphic ages in arctic Alaska in the vicinity of the Philip Smith Mountains quadrangle $\left(69^{\circ} \mathrm{N}, 150^{\circ} \mathrm{W}\right)$. We found overall greater exchangeable $\mathrm{K}$ concentrations, exchangeable and acid digestible $\mathrm{P}$ and $\mathrm{Ca}$ concentrations, acid digestible and total $\mathrm{Ca} / \mathrm{Na}$ and $\mathrm{Ca} / \mathrm{Sr}$, and lower acid digestible ${ }^{87} \mathrm{Sr} /{ }^{86} \mathrm{Sr}$ in permafrost than in active-layer mineral soil. Of the surfaces with similar parent material, stream and soil data suggest that weathering has progressively depleted calcium carbonate in the active layer with increasing surface age. Our results suggest that increasing thaw depth will lead to increasing carbonate and $\mathrm{Ca}$ supply to soils and streams, as well as spatially variable increases in P and $\mathrm{K}$ supply. Geochemical differences between active-layer soil and permafrost suggest the possibility of using stream geochemistry to detect changes in active-layer thickness in watersheds.
\end{abstract}

\section{Introduction}

Chemical weathering of minerals is an integral part of soil formation and is ecologically important as a source of nutrients such as phosphorus, potassium, and calcium (Schlesinger, 1997); silicate weathering is geologically important as a sink for atmospheric carbon in the long-term carbon cycle (Walker et al., 1981). It is important to understand mineral weathering and related soil and stream geochemistry in arctic regions because global climate change may cause changes in both the nature and extent of mineral weathering in these regions. Continuous permafrost in arctic regions restricts mineral weathering to the active (seasonally thawed) layer, and warming climate is likely to cause increased active-layer thickness (Anisimov et al., 1997). Increased active-layer thickness will allow minerals previously contained in permafrost to weather, thereby increasing the total mineral surface area that is exposed to weathering. Additionally, permafrost in some areas may contain very soluble minerals that largely have been removed by weathering in overlying thawed soils. Thawing and weathering of these minerals could cause potentially important changes in stream and soil geochemistry for years to come; the nature of these changes will depend on the spatial and temporal progression of permafrost degradation.

Much progress has been made toward understanding soil chemistry and mineral weathering on the North Slope of Alaska. Soils on the North Slope were classified and physically described in early work by Tedrow et al. (1958) and in more recent studies by Ping et al. (1998) and Munroe and Bockheim (2001). In an investigation of the soil chemistry differences between non-acidic and acidic tundra, Bockheim et al. (1998) analyzed soil exchangeable chemistry and other soil characteristics across the Kuparuk River Basin of the North Slope, an area that overlaps the study area of this paper and includes loess, till, and colluvium deposits of different ages. Their study found a relationship between non- acidic tundra soils and greater amounts of exchangeable base cations. Munroe and Bockheim (2001) found that among the four most recently deglaciated surfaces in this region, soil profile concentrations of weathering products (clay and silt) increased significantly with age, suggesting that even in areas where cryoturbation is common, surface age is a factor in mineral weathering and soil development.

Although cycling of biogeochemically important elements such as carbon and nitrogen in soils and catchments of the Alaskan Arctic has been the subject of many studies (e.g. Giblin et al., 1991; Nadelhoffer et al., 1991; Hobbie et al., 2002; Judd and Kling, 2002), the geochemistry of other elements including macroand micronutrients released by mineral weathering is less well known. Stutter and Billet (2003) described the stream and soil chemistry of a Swedish arctic region more recently glaciated than the study area of this paper, but with similar sedimentary bedrock (including sandstone and limestone units), and found the system to be dominated by carbonate dissolution. Kling et al. (1992) investigated the chemistry of lakes and rivers over a wide region of the Alaskan North Slope and interpreted the water chemistry in the region of their study to also be dominated by the products of carbonate dissolution and related to surface age.

Both terrestrial (Shaver and Chapin, 1995) and aquatic (Hobbie et al., 1999) ecosystems in this region are nutrient limited, either by $\mathrm{P}$ alone or by $\mathrm{N}$ and $\mathrm{P}$. Therefore, enhanced release of mineral nutrients via weathering of newly thawed minerals may have an important ecological impact. Hobbie et al. (1999) measured a twofold increase in $\mathrm{P}$ concentrations in a stream passing through glacial deposits disturbed by construction and predicted that weathering of thawed permafrost minerals may increase aquatic $\mathrm{P}$ availability, causing bottom-up trophic effects.

Because of the potential for a unique, permafrost-related geochemical response of arctic systems to climate change, it is 
important to understand the current mineral weathering processes and geochemical characteristics of these systems. This study describes the elemental chemistry of soils and streams in the central Alaskan North Slope, examines mineral weathering as a function of surface age, and addresses the geochemical implications of thaw depth increases brought about by climate change. We analyzed stream water and several chemical fractions of soil and bedrock, and used elemental and $\mathrm{Sr}$ isotope ratios to identify the mineral sources of weathering products. These results will serve to further our understanding of weathering in arctic regions, as well as provide baseline information for predicting how warming climate may influence the biogeochemistry of ecosystems.

\section{Study Area}

Field research was conducted on the eastern North Slope of arctic Alaska near the foothills of the Brooks Range (Fig. 1), primarily in the Philip Smith Mountains quadrangle $\left(69^{\circ} \mathrm{N}\right.$, $\left.150^{\circ} \mathrm{W}\right)$. The entire region is underlain by continuous permafrost (Brown and Krieg, 1983). For the years 1990-2000, the mean active-layer thickness at three sites within the study area (Happy Valley, Imnavait Creek, and Toolik Lake) ranged from 33 to $60 \mathrm{~cm}$ (Brown et al., 2002); the maximum thaw depth we observed during field work was $80 \mathrm{~cm}$. Mean annual air temperatures on this part of the North Slope range from $-5.9^{\circ} \mathrm{C}$ in the Brooks Range to $-12.8^{\circ} \mathrm{C}$ at Prudhoe Bay (Haugen, 1982). The dominant vegetation types include tussock tundra, wet sedge tundra, and riparian willow communities (Giblin et al., 1991). The landscape descends from the steep, rocky foothills of the Brooks Range to gently rolling tundra with poorly developed drainage and many kettle and thermokarst lakes.

North of the foothills, the landscape is developed on till and outwash formed during several glaciations of varying ages, during which glaciers moved northward from the Brooks Range (Hamilton, 1978; Brown and Krieg, 1983; Hamilton, 2003). The oldest and most northerly recognizable glacial deposits resulted from the Gunsight Mountain (GM) glaciation, which is inferred to have occurred in the late Tertiary based on the similarity of erosional features to Pliocene deposits south of the Brooks Range (Hamilton, 1994). The second oldest glacial deposits resulted from the Anaktuvuk River (AR) glaciation, which is assigned an early Pleistocene age by paleomagnetic analysis of overlying sediments (Hamilton, 1986) and correlations with other Alaskan glacial sequences (Hamilton, 1994). The next two younger glaciations are each broken up into separate units consisting of advances and readvances separated by interglacial periods. The Sagavanirktok River glaciation is dated broadly to the middle Pleistocene (780,000-125,000 years B.P.; Hamilton, 2003) based on paleomagnetic data and correlation to glaciations elsewhere in Alaska (Hamilton, 1994), and is separated into two units: Sagavanirktok River 1 (Sag1) and 2 (Sag2). At least five separate phases of the Itkillik glaciation have been identified (Hamilton, 2003), but for the purpose of simplification in this paper we use the three main phases initially described by Hamilton and Porter (1975) and used by Hamilton (1986): Itkillik I (It1), Itkillik II (It2), and the latest Itkillik II re-advance (It3). It1 deposits are beyond the range of radiocarbon dating $(>40 \mathrm{ka}$ ) but have an inferred age between 50 and $120 \mathrm{ka}$; radiocarbon dating suggests ages between approximately 11.5 and $24 \mathrm{ka}$ for It 2 deposits and $11.4 \mathrm{ka}$ and 12.8 for It 3 deposits (Hamilton, 1986, 2003). These surfaces have loess cover ranging from greater than $15 \mathrm{~m}$ on the oldest surfaces, through thin but still continuous cover on intermediate-aged surfaces, to

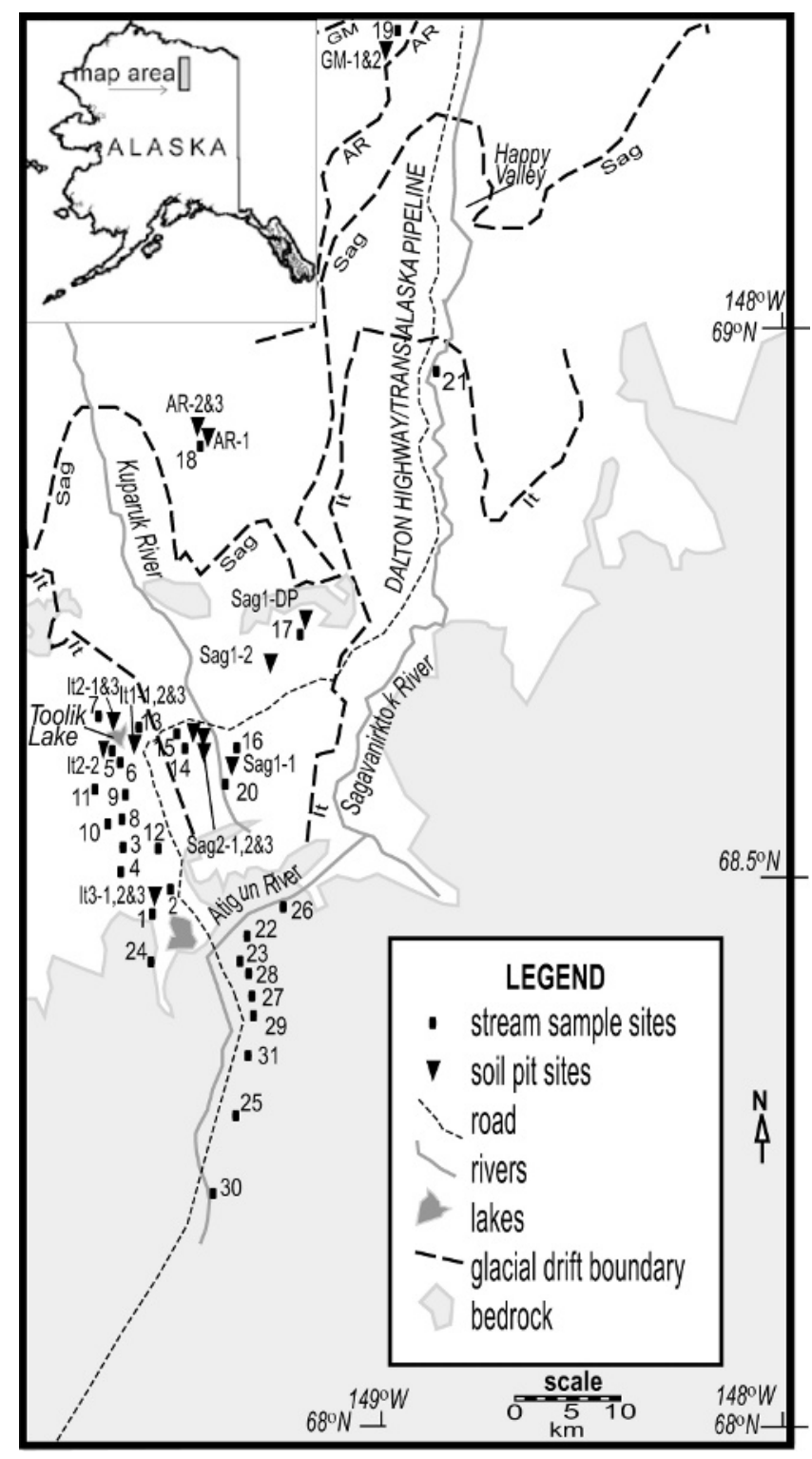

FIGURE 1. Map of study area with locations of soil (triangle) and stream (square) sampling sites. Glacial deposit and bedrock boundaries after Hamilton (1978, 2003), Mull and Adams (1985), and Brown and Krieg (1983).

loess-free moraine crests on the youngest surfaces (Hamilton, 1994, 2003).

The bedrock formations in the Brooks Range foothills, from which the glacial till is derived, consist primarily of conglomerates, sandstones, and limestones, with some shale and phyllite (Menzie et al., 1985; Mull and Adams, 1985). Based on the gentle topography of the youngest (It3) moraines, Hamilton (2003) inferred that they contain abundant fine sediments derived from large nearby lake beds and therefore may be compositionally different from other glacial deposits.

The soils developed on these deglaciated surfaces are classified broadly as Gelisols, and more specifically as Turbels and Orthels, depending on the amount of apparent cryoturbation (Munroe and Bockheim, 2001). In most areas, the mineral soil is overlain by a thick (10 or more centimeters) organic horizon. The soils are characterized by medium texture, poor drainage, and cryogenic features such as warped horizons and ice lenses (Ping et al., 1998). 


\section{Methods}

\section{WATER SAMPLING AND ANALYSIS}

We sampled small first- or second-order streams in watersheds entirely contained within each of the seven glacial surfaces of different ages in the study area, and within each of the major bedrock types in the foothills north of the Brooks Range (Fig. 1); we also sampled the major rivers (up to fourth order) in the study area. Precipitation samples were collected in acid-washed polyethylene containers. All water samples were filtered through $0.45 \mu \mathrm{m}$ polypropylene filters into acid-washed polyethylene bottles in the field, acidified with ultra-pure hydrochloric acid, and refrigerated at $<10^{\circ} \mathrm{C}$ until analysis.

All water samples were analyzed using a Perkin Elmer Optima 3300 DV Inductively Coupled Plasma Optical Emission Spectrometer (ICP-OES) using five- to eight-point calibration curves for element concentrations (including $\mathrm{Ba}, \mathrm{Ca}, \mathrm{Fe}, \mathrm{K}, \mathrm{Mg}$, $\mathrm{Na}, \mathrm{S}, \mathrm{Si}$, and $\mathrm{Sr}$ ). Two High-Purity ${ }^{\circledR}$ standards (Trace Metals in Drinking Water and River Sediment-B) and one in-house standard were used for quality control. These standards were analyzed to within $\pm 10 \%$ of the known values for $\mathrm{Fe}$ and $\mathrm{Sr} ; \pm 17 \%$ for $\mathrm{S}$, and $\pm 5 \%$ for all other elements.

\section{SOIL SAMPLING AND ANALYSIS}

During late July and August of 2002 and 2003, two to four soil sampling pits on each glacial deposit surface were excavated (Fig. 1), with the exception of the GM surface, on which a single large trench was excavated and sampled on opposite sides. Sites were randomly selected on flat (slope $<2^{\circ}$ ) moraine crests or shoulders. Vegetation at the pit sites indicated that all of the sites except one were located on moist acidic tundra; one of the It 2 pit sites was located on non-acidic tundra. Each pit was excavated through active-layer soil and at least several $\mathrm{cm}$ into frozen ground. Soil profiles and visible horizons were measured and described, and homogenized samples were taken from each visible horizon. We took 3-6 horizon samples per pit, usually consisting of layers designated "O" (peat), "A" (an oxidized layer with live roots), "B1" (the upper section of a thick gleyed layer), "B2" (the lower section of the same layer; this division was meant to split the thick "B" horizon approximately in half in order to look for differences with depth), "P1" (the first few centimeters of thickly laminated frozen soil, which may infrequently be part of the active layer), and "P2" (deeper, thinly laminated sediment in permafrost). Several soil profiles contained evidence of cryoturbation such as irregular horizon boundaries or buried peat inclusions. A deeper $(\sim 3 \mathrm{~m})$ permafrost sample (labeled Sag1-DP on Fig. 1 and in tables) was obtained from the Sag1 surface from the site of a thermokarst tundra collapse within a few days of that event.

Soil samples were dried at $35^{\circ} \mathrm{C}$, and mineral soils were sieved to separate the $<2-\mathrm{mm}$ size fraction, which was used for further analyses. We consider the $>2 \mathrm{~mm}$ fraction to contribute a negligible amount to overall mineral weathering of the soil due to its low ratio of surface area to volume; the $>2 \mathrm{~mm}$ fraction appeared to consist mostly of chert pebbles and quartzite cobbles and was on average less than $35 \%$ by mass of each sample. Percent organic matter was determined for samples from one representative pit on several surfaces by loss on ignition (LOI) via combustion at $450^{\circ} \mathrm{C}$ for $24 \mathrm{~h}$, following the method of Heiri et al (2001).

Following the methods of Blum et al. (2002), $0.5 \mathrm{~g}$ soil samples were leached and digested sequentially with $5 \mathrm{~mL}$ of each of the following ultra-pure solutions: (1) $1 \mathrm{M} \mathrm{NH}_{4} \mathrm{Cl}(\mathrm{pH}=7)$ at room temperature for $20 \mathrm{~h}$ to obtain the exchangeable fraction; (2) $1 \mathrm{M} \mathrm{HNO}_{3}$ at room temperature for $20 \mathrm{~h}$ to dissolve easily soluble minerals (which in these soils are mostly carbonates but include minor amounts of phosphate and some sulfides) and to leach remaining labile elements bound to organics; and (3) concentrated $\mathrm{HNO}_{3}$ at $150^{\circ} \mathrm{C}$ for $3 \mathrm{~h}$ to partially digest less soluble but still potentially weatherable minerals such as biotite, plagioclase, potassium feldspar, sulfides, and some oxides and clays. For convenience, we will refer to the solutions obtained via the second and third steps in the sequential digest procedure as the "cold acid digestible" and "hot acid digestible" fractions, respectively. While we aim to estimate "easily weatherable" and "less weatherable" fractions of the soil with these sequential digests, these digests are operationally defined and thus do not represent clearly defined reservoirs within the soil. Furthermore, while elemental $\mathrm{P}$ and $\mathrm{S}$ (possibly occurring as $\mathrm{PO}_{4}{ }^{3-}$ and $\mathrm{SO}_{4}{ }^{2-}$ ) released by $\mathrm{NH}_{4} \mathrm{Cl}$ are not necessarily "exchangeable," they represent the most labile fraction of those elements, and for convenience they are referred to as part of the exchangeable fraction.

To obtain a total digest of mineral soil samples, about $0.1 \mathrm{~g}$ of each sample was fused with $1 \mathrm{~g}$ of technical grade $\mathrm{LiBO}_{2}$ in a graphite crucible at $1100^{\circ} \mathrm{C}$, then dissolved in approximately $60 \mathrm{~mL}$ of trace metal grade $5 \% \mathrm{HNO}_{3}$ and filtered through $25-\mu \mathrm{m}$ pore size cellulose fiber paper. Two procedural blanks $\left(\mathrm{LiBO}_{2}\right.$ with no sample added) and two U.S. Geological Survey Geochemical Reference Standards were also digested.

The soil leachate and digest solutions were analyzed for elemental concentrations by ICP-OES using six- to nine-point calibration curves. Quality control standards included HighPurity ${ }^{\circledR}$ ICP-Stock Solution for Si and CRM Soil Solution A and Trace Metals in Drinking Water for other elements. For the sequential digest solutions, these standards were analyzed within $\pm 7 \%$ for $\mathrm{Ba}, \mathrm{Ca}, \mathrm{Fe}, \mathrm{K}, \mathrm{Na}$, and $\mathrm{Sr} ; \pm 12 \%$ for $\mathrm{Si}$, and $\pm 14 \%$ for $\mathrm{P}$ and $\mathrm{S}$ in the same concentration ranges as the samples. For the total digests, these standards plus an in-house standard were analyzed to within $\pm 7 \%$ for $\mathrm{Ba}, \mathrm{P}$, and $\mathrm{Si}$; and $\pm 12 \%$ for $\mathrm{Ca}, \mathrm{Fe}$, $\mathrm{K}, \mathrm{Na}, \mathrm{S}, \mathrm{Sr}$ in the same concentration ranges as the samples.

\section{BEDROCK SAMPLING AND ANALYSIS}

Samples of each major rock unit on the eastern North Slope (Philip Smith quadrangle) were collected during the summer of 2002. Where possible bedrock samples were collected from outcrops in the same watersheds in which streams were sampled.

Polished thin sections were made of each rock sample, and mineral constituents were identified visually using a petrographic microscope or by an energy dispersive spectrometer (EDS) on a Hitachi S3200N scanning electron microscope (SEM). Subsamples of each rock sample were pulverized in a tungsten carbide ring mill and then subjected to the same sequential leach and digest procedure described above. After the hot acid digest, the remaining sample was digested with a mixture of concentrated ultra-pure $\mathrm{HF}, \mathrm{HNO}_{3}$, and $\mathrm{HCl}$ until all sample was in solution. The leachate and digest solution was analyzed by ICP-OES as described above.

\section{SR ISOTOPIC ANALYSES}

$\mathrm{Sr}$ was separated from selected water samples and soil and rock leachate and digest solutions by eluting a subsample through Eichrom Sr-Spec resin in a quartz cation exchange column. Fifty to $100 \mathrm{ng}$ of $\mathrm{Sr}$ along with $1 \mu \mathrm{L}$ of $\mathrm{H}_{3} \mathrm{PO}_{4}$ were then loaded onto a tungsten filament with $\mathrm{Ta}_{2} \mathrm{O}_{5}$ powder matrix. ${ }^{87} \mathrm{Sr} /{ }^{86} \mathrm{Sr}$ was 
TABLE 1

Chemistry of local rock units likely incorporated into glacial till deposits. Formation names are defined in the text.

\begin{tabular}{|c|c|c|c|c|c|c|c|c|c|c|c|c|c|c|c|}
\hline $\begin{array}{l}\text { Fm. } \\
\text { name }\end{array}$ & Rock type & Fraction & $\begin{array}{c}\text { Ba } \\
(\mu \mathrm{mol} / \mathrm{g})\end{array}$ & $\begin{array}{c}\text { Ca } \\
(\mu \mathrm{mol} / \mathrm{g})\end{array}$ & $\begin{array}{c}\mathbf{F e} \\
(\mu \mathrm{mol} / \mathrm{g})\end{array}$ & $\begin{array}{c}\mathbf{K} \\
(\mu \mathrm{mol} / \mathrm{g})\end{array}$ & $\begin{array}{c}\text { Mg } \\
(\mu \mathrm{mol} / \mathrm{g})\end{array}$ & $\begin{array}{c}\mathbf{N a} \\
(\mu \mathrm{mol} / \mathrm{g})\end{array}$ & $\begin{array}{c}\mathbf{P} \\
(\mu \mathrm{mol} / \mathrm{g})\end{array}$ & $\begin{array}{c}\mathbf{S} \\
(\mu \mathrm{mol} / \mathrm{g})\end{array}$ & $\begin{array}{c}\mathbf{S i} \\
(\mu \mathrm{mol} / \mathrm{g})\end{array}$ & $\begin{array}{c}\mathbf{S r} \\
(\mu \mathrm{mol} / \mathrm{g})\end{array}$ & $\begin{array}{c}\mathbf{C a} / \mathbf{N a} \\
\text { (molar) }\end{array}$ & $\begin{array}{c}\mathbf{C a} / \mathbf{S r} \\
\text { (molar) }\end{array}$ & ${ }^{87} \mathrm{Sr} / /^{86} \mathrm{Sr}$ \\
\hline Dbs & phyllite/slate & exch. & 0.051 & 25.3 & 1.22 & 6.92 & 13.6 & 2.64 & $<0.09$ & 1.77 & 0.83 & 0.110 & 9.6 & 230 & 0.727052 \\
\hline Dke & ss/cong. & exch. & 0.031 & 2.94 & 0.63 & 9.68 & 4.12 & 1.59 & $<0.09$ & 0.15 & 0.30 & 0.020 & 1.8 & 147 & 0.723253 \\
\hline PMI & limestone & exch. & 0.009 & 132 & 0.28 & 1.19 & 2.63 & 0.40 & 0.23 & $<0.08$ & 0.36 & 0.092 & 326 & 1430 & 0.708986 \\
\hline $\mathrm{Pe}$ & shale & exch. & 1.35 & 30.9 & 1.28 & 12.7 & 10.9 & 0.53 & $<0.09$ & 0.42 & 4.79 & 0.097 & 58.7 & 319 & 0.715667 \\
\hline $\mathbf{K f}$ & sandstone & exch. & 2.57 & 11.9 & 0.14 & 20.4 & 19.4 & 4.50 & $<0.09$ & 0.40 & 0.64 & 0.052 & 2.6 & 227 & 0.717860 \\
\hline Kn & sandstone & exch. & 0.277 & 35.1 & 0.47 & 6.46 & 12.3 & 4.35 & $<0.09$ & 4.13 & $<0.06$ & 0.066 & 8.1 & 533 & 0.713092 \\
\hline Dbs & phyllite/slate & cold dig. & 0.016 & 660 & 348 & 4.17 & 314 & 0.62 & 20.4 & 54.6 & 60.4 & 0.831 & 1060 & 793 & 0.725383 \\
\hline Dke & ss/cong. & cold dig. & 0.085 & 12.3 & 67.5 & 6.12 & 6.73 & 0.47 & 2.88 & $<0.08$ & 39.2 & 0.029 & 26.3 & 425 & 0.719324 \\
\hline PMI & limestone & cold dig. & 0.044 & 5970 & 1.02 & 1.01 & 35.9 & 0.09 & 17.1 & $<0.08$ & 6.66 & 1.69 & 64000 & 3530 & 0.708058 \\
\hline $\mathrm{Pe}$ & shale & cold dig. & 2.97 & 55.4 & 80.6 & 8.29 & 71.3 & $<0.073$ & 2.01 & 1.74 & 44.6 & 0.103 & $>758$ & 540 & 0.714122 \\
\hline $\mathbf{K f}$ & sandstone & cold dig. & 2.49 & 45.7 & 141 & 12.5 & 97.5 & 3.12 & 21.2 & 2.71 & 97.3 & 0.096 & 14.7 & 477 & 0.712404 \\
\hline Kn & sandstone & cold dig. & 0.415 & 85.1 & 127 & 2.69 & 62.4 & $<0.073$ & 15.8 & 23.7 & 84.5 & 0.122 & $>1160$ & 695 & 0.713419 \\
\hline Dbs & phyllite/slate & hot dig. & $<0.001$ & 15.1 & 518 & 13.2 & 252 & $<0.073$ & $<0.09$ & 19.2 & 350 & 0.060 & $>207$ & 251 & 0.731134 \\
\hline Dke & ss/cong. & hot dig. & $<0.001$ & $<0.04$ & 229 & 20.1 & 8.04 & $<0.073$ & 2.30 & 0.15 & 150 & 0.060 & - & $<0.59$ & 0.723511 \\
\hline PMI & limestone & hot dig. & 0.019 & 534 & 2.38 & 1.19 & 2.17 & $<0.073$ & 1.37 & 0.18 & 14.0 & 0.099 & $>7310$ & 5380 & 0.713099 \\
\hline $\mathrm{Pe}$ & shale & hot dig. & 0.097 & $<0.04$ & 365 & 51.5 & 196 & $<0.073$ & $<0.09$ & 1.29 & 184 & 0.022 & - & $<1.60$ & 0.753962 \\
\hline $\mathbf{K f}$ & sandstone & hot dig. & 1.52 & $<0.04$ & 189 & 57.1 & 127 & 11.1 & 3.73 & 2.50 & 281 & 0.031 & $<0.003$ & $3<1.11$ & 0.735151 \\
\hline Kn & sandstone & hot dig. & 0.610 & 5.67 & 334 & 41.9 & 210 & 1.27 & 0.37 & 6.44 & 495 & 0.057 & 4.5 & 100 & 0.727727 \\
\hline Dbs & phyllite/slate & res. dig. & 2.66 & $<0.04$ & 91.3 & 452 & 1.32 & 290 & 0.28 & 1.48 & 1090 & 0.263 & $>0.0001$ & $<0.13$ & 0.740233 \\
\hline Dke & ss/cong. & res. dig. & $<0.001$ & $<0.04$ & 109 & 27.8 & 6.26 & $<0.073$ & 0.95 & $<0.08$ & 1560 & 0.208 & - & $<0.17$ & 0.714283 \\
\hline PMI & limestone & res. dig. & 0.035 & 1.23 & 0.17 & 0.50 & $<0.0001$ & $<0.073$ & $<0.09$ & 0.47 & 547 & 0.007 & $>16.4$ & 168 & 0.708846 \\
\hline $\mathrm{Pe}$ & shale & res. dig. & 1.22 & $<0.04$ & 259 & 583 & 41.2 & 91.3 & 0.22 & 1.25 & 780 & 0.412 & $<0.0003$ & $<0.085$ & 50.741406 \\
\hline $\mathbf{K f}$ & sandstone & res. dig. & 2.30 & $<0.04$ & 26.9 & 50.7 & 29.2 & 95.1 & 0.29 & 1.82 & 939 & 0.162 & $<0.0004$ & $<0.22$ & 0.715210 \\
\hline Kn & sandstone & res. dig. & 4.31 & 5.83 & 91.9 & 319 & 44.2 & 273 & 1.65 & 1.00 & 926 & 0.638 & 0.02 & 9.1 & 0.723082 \\
\hline
\end{tabular}

$\mathrm{PM} 1$ elemental data and cold digestible ${ }^{87} \mathrm{Sr} /{ }^{86} \mathrm{Sr}$ represent a mean of data from 5 samples of the PMl from different locations and members; other PM1 ${ }^{87} \mathrm{Sr} /{ }^{86} \mathrm{Sr}$ data and data for all other units are from one representative sample. Errors for elemental data and ${ }^{87} \mathrm{Sr} /{ }^{86} \mathrm{Sr}$ reported in text. Exch. $=$ exchangeable fraction; cold dig. $=$ cold acid digestible fraction; hot dig. = hot acid digestible fraction; res. dig. = residual (HF) digestible fraction.

determined by multiple-collector thermal ionization mass spectrometry (TIMS) on a Finnigan MAT 262. To correct for instrumental mass bias, ${ }^{86} \mathrm{Sr} /{ }^{88} \mathrm{Sr}$ was normalized to 0.1194 . Between 50 and 200 replicate ${ }^{87} \mathrm{Sr} /{ }^{86} \mathrm{Sr}$ ratios were measured for each sample. Internal precision $( \pm 2 \sigma)$ calculated from these replicates was generally less than \pm 0.000030 ; exceptions are noted in tables and graphs. The Sr standard NBS-987 was analyzed after every 12 samples with a mean value of $0.710231 \pm 0.000016(2 \sigma)$ during the period of sample analyses.

\section{Results}

\section{BEDROCK COMPOSITION AND GEOCHEMISTRY}

Based on the location and extent of rock unit outcrops in the eastern Brooks Range foothills and on cobbles found in soil pits by us and by Hamilton (2003), we identified six units (as mapped by Mull and Adams, 1985) likely to comprise the bulk of the glacial till in the study area: the Devonian Beaucoup phyllite (Dbs), the Devonian Ear Peak sandstone member of the Kanayut conglomerate and sandstone (Dke), the Permian-Mississippian Lisburne limestone (PMl), the Permian Echooka shale (Pe), the Cretaceous Fortress Mountain conglomerate and sandstone (Kf), and the Cretaceous Nanushuk sandstone (Kn). Because the Kn outcrops north of the Sagavanirktok River glacial deposits, it is likely included only in the older and more northerly Gunsight and Anaktuvuk glacial deposits.

Visual and SEM thin section analyses suggest that all three sandstones (Dke, Kf, and $\mathrm{Kn}$ ) are composed primarily of quartz and chert and contain minor amounts of biotite/vermiculite, muscovite, and oxide and sulfide minerals; the $\mathrm{Kf}$ and $\mathrm{Kn}$ also contain minor plagioclase and glauconite. The limestone unit (PMl) appeared chert-rich and argillaceous, which is consistent with the description of this unit by Moore et al. (1994).

The composition of sequential and bulk digests of these six units is reported in Table 1. As expected, the limestone had the lowest ${ }^{87} \mathrm{Sr} /{ }^{86} \mathrm{Sr}$ values and highest $\mathrm{Ca} / \mathrm{Sr}$ of the six units; the phyllite (Dbs) and the Devonian sandstone (Dke) had the highest exchangeable and cold acid digestible fraction ${ }^{87} \mathrm{Sr} /{ }^{86} \mathrm{Sr}$ values. The elemental ratios $\mathrm{Ca} / \mathrm{Na}$ and $\mathrm{Ca} / \mathrm{Sr}$ are often used as indicators of calcium carbonate contribution because calcium carbonate has high $\mathrm{Ca}$ but low $\mathrm{Na}$ and $\mathrm{Sr}$ concentrations, while the reverse is true for many common silicate minerals. Strontium isotope ratios can be used similarly because most carbonates have low ${ }^{87} \mathrm{Sr} /{ }^{86} \mathrm{Sr}$ while many silicate minerals have higher ${ }^{87} \mathrm{Sr} /{ }^{86} \mathrm{Sr}$ values (e.g. Palmer and Edmond, 1992). Because the carbonate and silicate rocks in this area follow these general patterns, we use the elemental concentration ratios $\mathrm{Ca} / \mathrm{Na}$ and $\mathrm{Ca} / \mathrm{Sr}$ and the isotope ratio ${ }^{87} \mathrm{Sr} /{ }^{86} \mathrm{Sr}$ as indicators of the calcium carbonate contribution to soil fractions and stream water solutes.

\section{SOIL GEOCHEMISTRY}

Geochemical characteristics of the soil exchangeable fraction, cold acid digestible fraction, hot acid digestible fraction, and total soil digests are reported in Tables 2-5. The It 2 surface has the highest exchangeable $\mathrm{Ca}$ concentrations, $\mathrm{Ca} / \mathrm{Na}$, and $\mathrm{Ca} / \mathrm{Sr}$ (Table 2). In the mineral soil of most profiles, there is a trend of increasing $\mathrm{Ca}$ concentrations and $\mathrm{Ca} / \mathrm{Sr}$ and decreasing ${ }^{87} \mathrm{Sr} /{ }^{86} \mathrm{Sr}$ with depth (Table 2). Statistical tests (ANOVA or Welch ANOVA where the variances are unequal) were performed on concentration 
TABLE 2

Mean exchangeable soil fraction (per gram dry soil). Surface and horizon names are defined in the text.

\begin{tabular}{|c|c|c|c|c|c|c|c|c|c|c|c|c|c|c|}
\hline Surface & Horizon & $\begin{array}{c}\text { Depth } \\
(\mathrm{cm})\end{array}$ & $\begin{array}{c}\mathbf{B a} \\
(\mu \mathrm{mol} / \mathrm{g})\end{array}$ & $\begin{array}{c}\text { Ca } \\
(\mu \mathrm{mol} / \mathrm{g})\end{array}$ & $\begin{array}{c}\mathbf{F e} \\
(\mu \mathrm{mol} / \mathrm{g})\end{array}$ & $\begin{array}{c}\mathbf{K} \\
(\mu \mathrm{mol} / \mathrm{g})\end{array}$ & $\begin{array}{c}\mathbf{N a} \\
(\mu \mathrm{mol} / \mathrm{g})\end{array}$ & $\begin{array}{c}\mathbf{P} \\
(\mu \mathrm{mol} / \mathrm{g})\end{array}$ & $\begin{array}{c}\mathbf{S} \\
(\mu \mathrm{mol} / \mathrm{g})\end{array}$ & $\begin{array}{c}\mathbf{S i} \\
(\mu \mathrm{mol} / \mathrm{g})\end{array}$ & $\begin{array}{c}\mathbf{S r} \\
(\mu \mathrm{mol} / \mathrm{g})\end{array}$ & $\begin{array}{c}\mathbf{C a} / \mathbf{N a} \\
\text { (molar) }\end{array}$ & $\begin{array}{c}\mathbf{C a} / \mathbf{S r} \\
\text { (molar) }\end{array}$ & ${ }^{87} \mathrm{Sr} /{ }^{86} \mathrm{Sr}^{* *}$ \\
\hline \multirow[t]{5}{*}{ It3 $(n=4)$} & $\mathrm{O}$ & $0-7$ & 0.163 & 92.0 & 3.31 & 7.12 & 0.22 & 0.58 & 0.91 & 2.49 & 0.087 & 420 & 1062 & 0.713660 \\
\hline & A & $7-19$ & 0.296 & 7.29 & 0.80 & 1.42 & 0.10 & 0.02 & 0.07 & 1.05 & 0.011 & 70.4 & 687 & $0.720245^{*}$ \\
\hline & B1 & 19-39 & 0.374 & 6.55 & 0.98 & 1.49 & 0.12 & 0.03 & 0.14 & 0.60 & 0.011 & 55.8 & 577 & 0.720483 \\
\hline & B2 & $39-60$ & 0.518 & 7.27 & 0.87 & 2.61 & 0.11 & 0.02 & 0.19 & 0.41 & 0.013 & 63.6 & 543 & 0.719548 \\
\hline & $\mathrm{P}$ & $62-71$ & 0.779 & 13.1 & 3.64 & 4.62 & 0.16 & 0.10 & 0.47 & 1.87 & 0.027 & 84.1 & 490 & 0.716431 \\
\hline \multirow[t]{5}{*}{ It2 $(n=3)$} & $\mathrm{O}$ & $0-16$ & 0.448 & 174 & 1.63 & 1.12 & 0.34 & 0.43 & 2.88 & 0.80 & 0.109 & 509 & 1596 & 0.709544 \\
\hline & A & $18-29$ & 0.700 & 52.7 & 2.31 & 0.81 & 0.11 & 0.12 & 0.11 & 1.75 & 0.041 & 489 & 1282 & 0.710457 \\
\hline & B1 & $29-46$ & 0.753 & 57.9 & 1.99 & 1.65 & 0.15 & 0.08 & 0.48 & 1.92 & 0.040 & 395 & 1450 & 0.709485 \\
\hline & $\mathrm{B} 2$ & $46-62$ & 0.428 & 83.3 & 3.95 & 2.14 & 0.20 & 0.15 & 2.82 & 3.05 & 0.060 & 425 & 1397 & $0.709496^{*}$ \\
\hline & $\mathrm{P}$ & $66-73$ & 0.302 & 88.5 & 1.85 & 2.47 & 0.55 & 0.10 & 4.74 & 1.12 & 0.071 & 162 & 1244 & 0.709585 \\
\hline \multirow[t]{6}{*}{ It1 $(n=3)$} & $\mathrm{O}^{* * *}$ & $0-7$ & 0.384 & 20.5 & 2.69 & 7.10 & 0.20 & 0.18 & 1.41 & 0.44 & 0.033 & 105 & 627 & $0.721412 *$ \\
\hline & A & $8-23$ & 0.296 & 4.71 & 1.03 & 0.64 & 0.06 & 0.02 & 0.12 & 0.31 & 0.007 & 78.1 & 657 & 0.718664 \\
\hline & $\mathrm{B} 1$ & $23-40$ & 0.373 & 5.48 & 0.60 & 0.69 & 0.06 & 0.04 & 0.11 & 0.22 & 0.009 & 93.2 & 642 & $0.718510^{*}$ \\
\hline & B2 & $35-49$ & 0.552 & 6.80 & 1.50 & 0.75 & 0.10 & 0.05 & 0.07 & 0.82 & 0.010 & 65.5 & 701 & 0.717530 \\
\hline & $\mathrm{P} 1$ & $49-60$ & 0.501 & 7.38 & 2.98 & 0.96 & 0.10 & 0.07 & 0.10 & 1.60 & 0.009 & 75.5 & 797 & 0.715944 \\
\hline & $\mathrm{P} 2$ & $62-69$ & 0.489 & 34.7 & 0.42 & 1.76 & 0.10 & 0.04 & 0.14 & 0.28 & 0.037 & 359 & 951 & 0.711278 \\
\hline \multirow[t]{6}{*}{ Sag2 $(n=3)$} & $\mathrm{O}$ & $0-8$ & 0.242 & 32.0 & 2.72 & 6.55 & 0.34 & 0.68 & 1.49 & 0.91 & 0.048 & 93.7 & 670 & 0.716872 \\
\hline & A & $8-25$ & 0.409 & 4.77 & 0.58 & 0.85 & 0.10 & 0.05 & 0.15 & 0.35 & 0.009 & 47.9 & 541 & 0.720050 \\
\hline & B1 & $25-42$ & 0.861 & 14.2 & 1.93 & 1.43 & 0.23 & 0.06 & 0.09 & 1.52 & 0.023 & 61.8 & 612 & $0.720851^{*}$ \\
\hline & B2 & $38-55$ & 0.365 & 3.05 & 1.52 & 0.66 & 0.15 & 0.04 & 0.11 & 1.33 & 0.006 & 19.9 & 504 & 0.717221 \\
\hline & $\mathrm{P} 1$ & $55-69$ & 0.495 & 5.39 & 0.95 & 1.24 & 0.15 & 0.02 & 0.08 & 0.49 & 0.011 & 35.2 & 486 & 0.715970 \\
\hline & $\mathrm{P} 2$ & $71-75$ & 0.861 & 21.5 & 1.41 & 2.43 & 0.20 & 0.06 & 0.14 & 0.78 & 0.030 & 105 & 709 & $0.716069^{*}$ \\
\hline \multirow[t]{5}{*}{ Sag1 $(n=2)$} & $\mathrm{O}$ & $0-13$ & 0.543 & 30.6 & 3.13 & 3.56 & 0.29 & 0.21 & 1.18 & 0.65 & 0.044 & 106 & 696 & 0.717314 \\
\hline & A & $13-22$ & 0.611 & 12.3 & 1.76 & 1.22 & 0.14 & 0.08 & 0.34 & 1.20 & 0.018 & 88.0 & 668 & 0.717041 \\
\hline & B1 & $22-40$ & 0.580 & 5.89 & 0.45 & 0.82 & 0.15 & 0.04 & 0.10 & 0.37 & 0.009 & 39.0 & 635 & 0.719167 \\
\hline & B2 & $35-50$ & 0.727 & 7.17 & 0.85 & 1.29 & 0.16 & 0.03 & 0.15 & 0.54 & 0.011 & 44.3 & 630 & $0.717241^{*}$ \\
\hline & $\mathrm{P}$ & $50-58$ & 0.680 & 7.24 & 2.85 & 1.62 & 0.17 & 0.07 & 0.20 & 1.84 & 0.011 & 41.5 & 656 & $0.716745^{*}$ \\
\hline Sag1-DP & $\mathrm{P}$ (deep) & $200-300$ & 0.340 & 79.4 & 0.74 & 1.74 & $<0.04$ & 0.10 & 5.88 & 1.85 & 0.102 & $>1980$ & 780 & 0.713109 \\
\hline \multirow[t]{6}{*}{$\operatorname{AR}(n=3)$} & $\mathrm{O}$ & $0-11$ & 0.116 & 68.6 & 0.78 & 4.56 & 0.26 & 0.87 & 1.20 & 0.48 & 0.070 & 269 & 980 & 0.708721 \\
\hline & A & $11-27$ & 0.458 & 67.1 & 3.29 & 1.34 & 0.21 & 0.19 & 0.35 & 2.10 & 0.076 & 322 & 880 & 0.708773 \\
\hline & $\mathrm{B} 1$ & $27-50$ & 0.549 & 73.7 & 1.38 & 1.38 & 0.23 & 0.09 & 0.06 & 1.94 & 0.080 & 318 & 925 & 0.708416 \\
\hline & B2 & $45-75$ & 0.616 & 78.2 & 1.89 & 1.50 & 0.21 & 0.12 & 0.00 & 1.61 & 0.084 & 367 & 931 & $0.708313^{*}$ \\
\hline & $\mathrm{P} 1$ & $70-80$ & 0.691 & 75.7 & 1.29 & 1.56 & 0.27 & 0.07 & 0.08 & 0.87 & 0.075 & 282 & 1010 & 0.708253 \\
\hline & $\mathrm{P} 2$ & $85-93$ & 0.688 & 68.6 & 4.42 & 1.55 & 0.19 & 0.15 & 0.01 & 2.76 & 0.073 & 356 & 942 & 0.708296 \\
\hline \multirow[t]{7}{*}{ GM $(n=2)$} & $\mathrm{Oe}$ & $0-13$ & 0.185 & 38.5 & 1.87 & 5.93 & 0.31 & 0.73 & 0.86 & 0.77 & 0.053 & 125 & 731 & $0.710621^{*}$ \\
\hline & $\mathrm{B} 1$ & $13-35$ & 0.755 & 28.5 & 1.95 & 1.10 & 0.39 & 0.02 & 0.24 & 0.61 & 0.042 & 73.1 & 673 & $0.711741^{*}$ \\
\hline & B2 & $38-44$ & 0.828 & 39.0 & 5.19 & 1.17 & 0.42 & 0.08 & 0.20 & 3.16 & 0.057 & 92.2 & 690 & 0.710258 \\
\hline & IIOa & $44-55$ & 0.431 & 105 & 3.16 & 1.04 & 0.26 & 0.23 & 1.21 & 0.76 & 0.112 & 405 & 937 & 0.710080 \\
\hline & $\mathrm{P} 1$ & $50-54$ & 0.327 & 82.8 & 3.23 & 1.11 & 0.19 & 0.23 & 0.88 & 2.17 & 0.099 & 425 & 833 & $0.709814^{*}$ \\
\hline & IIB & $57-90$ & 0.278 & 150 & 2.28 & 1.93 & 0.36 & 0.16 & 0.74 & 1.64 & 0.145 & 421 & 1040 & 0.709412 \\
\hline & $\mathrm{P} 2$ & $100-110$ & 0.289 & 85.0 & 0.59 & 1.90 & 0.73 & 0.05 & 1.61 & 0.59 & 0.155 & 116 & 548 & 0.710615 \\
\hline
\end{tabular}

Errors for elemental data and ${ }^{87} \mathrm{Sr} /{ }^{86} \mathrm{Sr}$ as reported in text, with the following exceptions:

* Error $(2 \sigma)$ greater than 0.000030 but less than 0.000075

** $n=1$ for all ${ }^{87} \mathrm{Sr} /{ }^{86} \mathrm{Sr}$ data.

*** $n=2$ for It1 O horizon.

data for select elements that were log-transformed to induce normal distribution. Although data sets for individual surfaces are not large enough to test reliably, statistical analyses of data from all surfaces combined show some significant $(p<0.05)$ differences between permafrost and active-layer mineral soil chemistry: exchangeable fraction $\mathrm{Ca}, \mathrm{K}$, and $\mathrm{P}$ concentrations are significantly greater in the permafrost (all "P" horizons) than in activelayer mineral soil (all "A" and "B" horizons). Despite these overall statistically significant differences, the magnitudes of within-surface differences between permafrost and active-layer mineral soil exchangeable fraction elemental concentrations vary substantially among surfaces (Table 2).
The cold acid digestible fractions of the It2, AR, and GM soil profiles have greater $\mathrm{Ca}$ concentrations and $\mathrm{Ca} / \mathrm{Sr}$ and lower ${ }^{87} \mathrm{Sr} /{ }^{86} \mathrm{Sr}$ than those of the other surfaces (Table 3 ). The cold acid digestible fraction of the mineral soil shows a general pattern across all surfaces of increasing $\mathrm{Ca}$ concentrations and $\mathrm{Ca} / \mathrm{Sr}$ and decreasing ${ }^{87} \mathrm{Sr} /{ }^{86} \mathrm{Sr}$ with depth, and on some surfaces P concentrations also increase with depth. Both $\mathrm{Ca}$ and $\mathrm{P}$ cold acid digestible fraction concentrations are significantly $(p \leq 0.05)$ greater in the permafrost than in the active-layer mineral soil when data from all surfaces are analyzed, and the differences in cold acid digestible fraction $\mathrm{K}$ concentrations are nearly significant $(p=0.06)$. These differences between active-layer mineral soil and permafrost vary by 
TABLE 3

Mean cold acid digestible soil fraction (per gram dry soil). Surface and horizon names are defined in the text.

\begin{tabular}{|c|c|c|c|c|c|c|c|c|c|c|c|c|c|c|}
\hline Surface & Horizon & $\begin{array}{l}\text { Depth } \\
(\mathrm{cm})\end{array}$ & $\begin{array}{c}\text { Ba } \\
(\mu \mathrm{mol} / \mathrm{g})\end{array}$ & $\begin{array}{c}\text { Ca } \\
(\mu \mathrm{mol} / \mathrm{g})\end{array}$ & $\begin{array}{c}\mathbf{F e} \\
(\mu \mathrm{mol} / \mathrm{g})\end{array}$ & $\begin{array}{c}\mathbf{K} \\
(\mu \mathrm{mol} / \mathrm{g})\end{array}$ & $\begin{array}{c}\mathbf{N a} \\
(\mu \mathrm{mol} / \mathrm{g})\end{array}$ & $\begin{array}{c}\mathbf{P} \\
(\mu \mathrm{mol} / \mathrm{g})\end{array}$ & $\begin{array}{c}\mathbf{S} \\
(\mu \mathrm{mol} / \mathrm{g})\end{array}$ & $\begin{array}{c}\mathbf{S i} \\
(\mu \mathrm{mol} / \mathrm{g})\end{array}$ & $\begin{array}{c}\mathbf{S r} \\
(\mu \mathrm{mol} / \mathrm{g})\end{array}$ & $\begin{array}{c}\mathbf{C a} / \mathbf{N a} \\
\text { (molar) }\end{array}$ & $\begin{array}{c}\mathbf{C a} / \mathbf{S r} \\
\text { (molar) }\end{array}$ & ${ }^{87} \mathrm{Sr} /{ }^{86} \mathrm{Sr} *$ \\
\hline \multirow[t]{5}{*}{ It3 $(n=4)$} & $\mathrm{O}$ & $0-7$ & 0.682 & 183 & 57.3 & 3.97 & 0.45 & 5.12 & 0.58 & 29.4 & 0.144 & 405 & 1270 & 0.713346 \\
\hline & A & $7-19$ & 0.123 & 7.77 & 147 & 1.41 & 0.29 & 2.06 & 0.68 & 42.1 & 0.011 & 26.7 & 681 & 0.713351 \\
\hline & B1 & 19-39 & 0.191 & 8.54 & 173 & 1.46 & 0.28 & 1.82 & 0.58 & 44.7 & 0.013 & 30.6 & 660 & 0.714043 \\
\hline & B2 & $39-60$ & 0.189 & 8.76 & 157 & 1.80 & 0.28 & 3.05 & 0.75 & 44.7 & 0.013 & 31.4 & 672 & 0.713707 \\
\hline & $\mathrm{P}$ & $62-71$ & 0.399 & 12.6 & 285 & 2.46 & 0.44 & 8.31 & 1.25 & 51.7 & 0.014 & 28.9 & 875 & 0.713848 \\
\hline \multirow[t]{5}{*}{ It2 $(n=3)$} & $\mathrm{O}$ & $0-16$ & 2.17 & 360 & 53.8 & 1.01 & 0.56 & 4.70 & 5.90 & 11.9 & 0.218 & 646 & 1650 & 0.710522 \\
\hline & A & $18-29$ & 1.09 & 60.7 & 119 & 1.35 & 0.39 & 11.3 & 2.47 & 59.8 & 0.070 & 156 & 864 & 0.710246 \\
\hline & B1 & $29-46$ & 1.34 & 62.4 & 122 & 1.79 & 0.46 & 11.2 & 4.05 & 68.8 & 0.072 & 136 & 869 & 0.709875 \\
\hline & B2 & $46-62$ & 2.17 & 122 & 117 & 1.99 & 0.41 & 12.2 & 7.12 & 72.2 & 0.118 & 297 & 1040 & 0.709850 \\
\hline & $\mathrm{P}$ & $66-73$ & 2.39 & 257 & 125 & 1.97 & 0.42 & 9.76 & 4.01 & 66.9 & 0.195 & 610 & 1320 & 0.709610 \\
\hline \multirow[t]{6}{*}{ It1 $(n=3)$} & $\mathrm{O}^{*}$ & $0-7$ & 0.350 & 10.4 & 68.1 & 3.64 & 0.18 & 3.73 & 1.19 & 18.8 & 0.017 & 59.3 & 627 & 0.720647 \\
\hline & A & $8-23$ & 0.152 & 6.03 & 125 & 0.95 & 0.10 & 2.04 & 0.34 & 28.5 & 0.009 & 62.5 & 702 & 0.713331 \\
\hline & B1 & $23-40$ & 0.180 & 8.67 & 121 & 1.04 & 0.10 & 3.23 & 0.39 & 32.1 & 0.012 & 88.3 & 729 & 0.712963 \\
\hline & B2 & $35-49$ & 0.186 & 7.23 & 124 & 1.14 & 0.13 & 3.10 & 0.19 & 39.2 & 0.011 & 57.6 & 658 & 0.712858 \\
\hline & P1 & $49-60$ & 0.136 & 6.28 & 95.7 & 1.11 & 0.13 & 2.41 & 0.31 & 31.9 & 0.009 & 47.8 & 685 & 0.712326 \\
\hline & $\mathrm{P} 2$ & $62-69$ & 0.423 & 79.8 & 125 & 1.40 & 0.09 & 5.60 & 0.64 & 42.2 & 0.052 & 929 & 1540 & 0.710896 \\
\hline \multirow[t]{6}{*}{ Sag2 $(n=3)$} & $\mathrm{O}$ & $0-8$ & 0.537 & 28.1 & 52.1 & 2.91 & 0.44 & 5.93 & 1.33 & 13.2 & 0.039 & 64.6 & 714 & 0.715214 \\
\hline & A & $8-25$ & 0.182 & 6.84 & 92.0 & 1.01 & 0.13 & 2.36 & 0.33 & 31.1 & 0.009 & 50.9 & 758 & 0.712909 \\
\hline & B1 & $25-42$ & 0.414 & 7.99 & 138 & 1.52 & 0.14 & 2.71 & 0.86 & 45.1 & 0.011 & 57.5 & 703 & 0.713031 \\
\hline & B2 & $38-55$ & 0.079 & 4.04 & 60.5 & 0.94 & 0.18 & 3.05 & 0.15 & 26.9 & 0.006 & 22.3 & 672 & 0.712395 \\
\hline & P1 & $55-69$ & 0.128 & 5.99 & 65.4 & 1.25 & 0.18 & 2.44 & 0.38 & 28.1 & 0.009 & 33.1 & 655 & 0.712871 \\
\hline & $\mathrm{P} 2$ & $71-75$ & 0.493 & 12.2 & 95.4 & 1.57 & 0.11 & 2.18 & 0.70 & 37.0 & 0.015 & 114 & 807 & 0.712010 \\
\hline \multirow[t]{5}{*}{ Sag1 $(n=2)$} & $\mathrm{O}$ & $0-13$ & 0.677 & 22.6 & 85.8 & 3.28 & 0.40 & 3.03 & 1.56 & 23.2 & 0.030 & 57.1 & 749 & 0.715044 \\
\hline & $\mathrm{A}$ & $13-22$ & 0.272 & 4.0 & 133 & 1.29 & 0.23 & 1.54 & 0.66 & 34.4 & 0.006 & 17.0 & 669 & 0.713519 \\
\hline & B1 & $22-40$ & 0.147 & 2.65 & 104 & 1.26 & 0.26 & 1.36 & 0.28 & 35.9 & 0.004 & 10.1 & 649 & 0.713252 \\
\hline & B2 & $35-50$ & 0.186 & 3.29 & 115 & 1.34 & 0.27 & 1.84 & 0.34 & 39.1 & 0.005 & 12.0 & 602 & 0.713607 \\
\hline & $\mathrm{P}$ & $50-58$ & 0.235 & 6.16 & 166 & 1.53 & 0.31 & 4.38 & 0.45 & 41.4 & 0.008 & 20.0 & 738 & 0.713144 \\
\hline Sag1-DP & $\mathrm{P}$ (deep) & $200-300$ & 1.75 & 244 & 236 & 1.46 & 18.8 & 21.5 & 9.08 & 169 & 0.279 & 13.0 & 874 & 0.711083 \\
\hline \multirow[t]{6}{*}{$\mathrm{AR}(n=3)$} & $\mathrm{O}$ & $0-11$ & 0.391 & 166 & 37.1 & 4.89 & 0.40 & 6.33 & 1.82 & 16.3 & 0.154 & 418 & 1080 & 0.708762 \\
\hline & A & $11-27$ & 0.727 & 82.7 & 171 & 1.97 & 0.73 & 19.7 & 1.66 & 65.2 & 0.164 & 113 & 503 & 0.709060 \\
\hline & B1 & $27-50$ & 0.726 & 183 & 137 & 2.37 & 0.63 & 20.3 & 0.90 & 85.9 & 0.213 & 288 & 857 & 0.708580 \\
\hline & B2 & $45-75$ & 0.446 & 280 & 70.8 & 2.16 & 0.49 & 13.2 & 0.01 & 66.4 & 0.250 & 573 & 1120 & 0.708235 \\
\hline & $\mathrm{P} 1$ & $70-80$ & 0.777 & 423 & 142 & 2.59 & 0.80 & 20.8 & 0.12 & 92.8 & 0.353 & 530 & 1200 & 0.708352 \\
\hline & $\mathrm{P} 2$ & $85-93$ & 0.740 & 320 & 94.2 & 2.45 & 0.58 & 19.0 & 0.01 & 84.3 & 0.309 & 549 & 1040 & 0.708425 \\
\hline \multirow[t]{7}{*}{ GM $(n=2)$} & Oe & $0-13$ & 0.394 & 54.8 & 29.4 & 6.32 & 0.34 & 5.52 & 1.21 & 7.06 & 0.075 & 160 & 729 & 0.710627 \\
\hline & B1 & $13-35$ & 0.310 & 14.1 & 162 & 3.18 & 1.21 & 4.66 & 3.19 & 62.6 & 0.029 & 11.6 & 490 & 0.711870 \\
\hline & B2 & $38-44$ & 0.467 & 18.3 & 286 & 1.86 & 0.39 & 3.20 & 1.72 & 57.0 & 0.034 & 47.3 & 538 & 0.711035 \\
\hline & IIOa & $44-55$ & 1.10 & 186 & 241 & 3.73 & 1.24 & 7.52 & 5.48 & 44.0 & 0.219 & 150 & 849 & 0.710836 \\
\hline & $\mathrm{P} 1$ & $50-54$ & 0.811 & 101 & 131 & 1.96 & 0.34 & 6.93 & 2.43 & 36.7 & 0.132 & 295 & 762 & 0.709522 \\
\hline & IIB & $57-90$ & 1.05 & 342 & 139 & 4.22 & 1.34 & 9.92 & 3.85 & 64.0 & 0.313 & 255 & 1090 & 0.709681 \\
\hline & $\mathrm{P} 2$ & $100-110$ & 1.07 & 1220 & 177 & 3.21 & 1.37 & 13.9 & 2.55 & 72.4 & 0.850 & 891 & 1440 & 0.709076 \\
\hline
\end{tabular}

Errors for elemental data and ${ }^{87} \mathrm{Sr} /{ }^{86} \mathrm{Sr}$ as reported in text; maximum error $(2 \sigma)$ for any ${ }^{87} \mathrm{Sr} /{ }^{86} \mathrm{Sr}$ value used in calculating means is 0.000068

* For ${ }^{87} \mathrm{Sr} /{ }^{86} \mathrm{Sr}, n=3$ for It $3, n=1$ for AR and GM; for other surfaces, $n$ is as reported.

surface: all surfaces have higher permafrost concentrations of $\mathrm{Ca}$; all surfaces except the GM and It2 have higher permafrost concentrations of $\mathrm{K}$; and cold acid digestible fraction $\mathrm{P}$ concentrations are higher in the permafrost on four of the surfaces.

The hot acid digestible fractions of mineral soil horizons on all surfaces contain some $\mathrm{Ca}$, but have lower $\mathrm{Ca} / \mathrm{Sr}$ and higher ${ }^{87} \mathrm{Sr} /{ }^{86} \mathrm{Sr}$ than either the exchangeable or cold acid digestible fractions (Table 4). This suggests that the majority of carbonate was dissolved before this step in the sequential digest procedure and that the $\mathrm{Ca}$ in this fraction is largely the result of silicate mineral dissolution.

The total digests of the mineral soil (Table 5) show that $\mathrm{Ca}$ generally increases with depth on each surface. The It2, AR, and $\mathrm{GM}$ surfaces have the highest $\mathrm{Ca}$ concentrations. On average, $\mathrm{SiO}_{2}$ comprises approximately $80 \%$ of the total mineral soil mass.
For a few samples, elemental concentrations from the acid digests sum to slightly more than the total digest concentration. These values are within analytical errors propagated through the sum in all except for the Ca values for the Sag1 deep permafrost sample. This discrepancy is likely the result of sample heterogeneity.

\section{STREAM GEOCHEMISTRY}

In streams on glacial deposit surfaces, dissolved Ca concentrations ranged from $12 \mu \mathrm{mol} \mathrm{L}{ }^{-1}$ on the Sag1 surface to $594 \mu \mathrm{mol} \mathrm{L}^{-1}$ on the It 2 surface. Streams draining only limestone bedrock had $\mathrm{Ca}$ concentrations up to $1606 \mu \mathrm{mol} \mathrm{L}^{-1}$ (Table 6). Dissolved $\mathrm{P}$ concentrations were generally less than $1 \mu \mathrm{mol} \mathrm{L}{ }^{-1}$, but several streams on the It 2 surface had concentrations greater than $10 \mu \mathrm{mol} \mathrm{L}{ }^{-1}$. Dissolved Si concentrations were less than 
TABLE 4

Mean hot acid digestible soil fraction (per gram dry soil). Surface and horizon names are defined in the text.

\begin{tabular}{|c|c|c|c|c|c|c|c|c|c|c|c|c|c|c|}
\hline Surface & Horizon & $\begin{array}{l}\text { Depth } \\
(\mathrm{cm})\end{array}$ & $\begin{array}{c}\mathbf{B a} \\
(\mu \mathrm{mol} / \mathrm{g})\end{array}$ & $\begin{array}{c}\text { Ca } \\
(\mu \mathrm{mol} / \mathrm{g})\end{array}$ & $\begin{array}{c}\mathbf{F e} \\
(\mu \mathrm{mol} / \mathrm{g})\end{array}$ & $\begin{array}{c}\mathbf{K} \\
(\mu \mathrm{mol} / \mathrm{g})\end{array}$ & $\begin{array}{c}\mathbf{N a} \\
(\mu \mathrm{mol} / \mathrm{g})\end{array}$ & $\begin{array}{c}\mathbf{P} \\
(\mu \mathrm{mol} / \mathrm{g})\end{array}$ & $\begin{array}{c}\mathbf{S} \\
(\mu \mathrm{mol} / \mathrm{g})\end{array}$ & $\begin{array}{c}\text { Si } \\
(\mu \mathrm{mol} / \mathrm{g})\end{array}$ & $\begin{array}{c}\mathbf{S r} \\
(\mu \mathrm{mol} / \mathrm{g})\end{array}$ & $\begin{array}{r}\mathbf{C a} / \mathbf{N a} \\
\text { (molar) }\end{array}$ & $\begin{array}{c}\mathbf{C a} / \mathbf{S r} \\
\text { (molar) }\end{array}$ & ${ }^{87} \mathrm{Sr} /{ }^{86} \mathrm{Sr}^{* * *}$ \\
\hline \multirow[t]{5}{*}{ It3 $(n=4)$} & $\mathrm{O}$ & $0-7$ & 0.309 & 51.2 & 196 & 5.42 & 16.7 & 13.0 & 26.2 & 10.5 & 0.045 & 3.07 & 1150 & 0.714412 \\
\hline & A & $7-19$ & 0.125 & 2.44 & 359 & 8.40 & 12.9 & 5.56 & 3.09 & 49.3 & 0.015 & 0.19 & 168 & 0.731134 \\
\hline & B1 & 19-39 & 0.145 & 2.37 & 409 & 10.1 & 13.0 & 6.74 & 3.77 & 44.2 & 0.014 & 0.18 & 173 & 0.732188 \\
\hline & B2 & $39-60$ & 0.183 & 2.17 & 441 & 14.3 & 12.9 & 8.67 & 5.75 & 68.2 & 0.019 & 0.17 & 113 & $0.733771 *$ \\
\hline & $\mathrm{P}$ & $62-71$ & 0.253 & 4.38 & 263 & 9.05 & 17.0 & 7.22 & 10.6 & 4.12 & 0.010 & 0.26 & 436 & 0.730624 \\
\hline \multirow[t]{5}{*}{ It2 $(n=3)$} & $\mathrm{O}$ & $0-16$ & 0.949 & 87.9 & 95.1 & .78 & 16.7 & 15.4 & 101 & 9.41 & 0.056 & 5.27 & 1570 & $0.709652 *$ \\
\hline & A & $18-29$ & 0.284 & 3.91 & 298 & 5.22 & 11.5 & 6.81 & 13.6 & 87.8 & 0.009 & 0.34 & 429 & 0.720106 \\
\hline & B1 & $29-46$ & 0.342 & 4.10 & 342 & 7.61 & 11.5 & 4.55 & 14.6 & 102 & 0.012 & 0.36 & 340 & $0.720671 *$ \\
\hline & B2 & $46-62$ & 0.353 & 7.20 & 341 & 10.5 & 11.2 & 7.20 & 24.5 & 47.9 & 0.015 & 0.64 & 485 & 0.725481 \\
\hline & $\mathrm{P}$ & $66-73$ & 0.324 & 13.2 & 395 & 4.94 & 8.34 & 8.20 & 27.1 & 50.9 & 0.019 & 1.58 & 710 & 0.715267 \\
\hline \multirow[t]{6}{*}{ It1 $(n=3)$} & $\mathrm{O} * * * *$ & $0-7$ & 0.296 & 6.06 & 317 & 9.33 & 17.2 & 23.2 & 25.9 & 15.4 & 0.012 & 0.35 & 526 & 0.724344 \\
\hline & A & $8-23$ & 0.107 & 1.76 & 330 & 5.85 & 12.1 & 5.35 & 2.54 & 60.4 & 0.008 & 0.15 & 231 & $0.727602 *$ \\
\hline & $\mathrm{B} 1$ & $23-40$ & 0.103 & 1.69 & 285 & 6.82 & 12.5 & 4.53 & 2.45 & 81.9 & 0.009 & 0.14 & 191 & 0.730939 \\
\hline & B2 & $35-49$ & 0.191 & 2.46 & 399 & 7.38 & 18.4 & 8.26 & 2.41 & 3.19 & 0.010 & 0.13 & 254 & 0.731677 \\
\hline & $\mathrm{P} 1$ & $49-60$ & 0.192 & 2.70 & 394 & 8.64 & 17.9 & 7.61 & 1.98 & 4.57 & 0.010 & 0.15 & 275 & 0.732349 \\
\hline & $\mathrm{P} 2$ & $62-69$ & 0.160 & 2.77 & 411 & 15.0 & 12.0 & 6.70 & 8.16 & 114 & 0.017 & 0.23 & 163 & 0.733956 \\
\hline \multirow[t]{6}{*}{ Sag2 $(n=3)$} & $\mathrm{O}$ & $0-8$ & 0.238 & 11.8 & 156 & 5.12 & 17.5 & 17.5 & 23.9 & 8.03 & 0.016 & 0.67 & 752 & $0.720922 *$ \\
\hline & A & $8-25$ & 0.126 & 2.14 & 283 & 5.86 & 12.0 & 5.98 & 3.10 & 45.3 & 0.007 & 0.18 & 295 & 0.722058 \\
\hline & B1 & $25-42$ & 0.231 & 2.38 & 343 & 9.99 & 11.9 & 7.13 & 6.14 & 54.2 & 0.012 & 0.20 & 190 & 0.722017 \\
\hline & B2 & $38-55$ & 0.187 & 3.45 & 216 & 4.52 & 18.3 & 2.95 & 1.38 & 4.15 & 0.009 & 0.19 & 394 & 0.723151 \\
\hline & P1 & $55-69$ & 0.191 & 3.08 & 386 & 4.25 & 17.5 & 7.44 & 3.01 & 4.87 & 0.007 & 0.18 & 465 & $0.721817^{*}$ \\
\hline & $\mathrm{P} 2$ & $71-75$ & 0.209 & 2.10 & 386 & 8.62 & 12.0 & 5.38 & 5.55 & 67.8 & 0.010 & 0.17 & 206 & 0.723476 \\
\hline \multirow[t]{5}{*}{ Sag1 $(n=2)$} & $\mathrm{O}$ & $0-13$ & 0.363 & 7.92 & 176 & 8.86 & 17.1 & 21.5 & 27.5 & 2.43 & 0.019 & 0.46 & 409 & $0.722149 * *$ \\
\hline & A & $13-22$ & 0.307 & 4.20 & 459 & 10.1 & 18.2 & 12.1 & 6.59 & 4.87 & 0.018 & 0.23 & 227 & 0.723068 \\
\hline & B1 & $22-40$ & 0.247 & 4.30 & 364 & 7.33 & 17.8 & 7.31 & 1.91 & 4.88 & 0.016 & 0.24 & 274 & $0.722645^{* *}$ \\
\hline & B2 & $35-50$ & 0.248 & 3.88 & 371 & 7.76 & 18.4 & 7.16 & 1.56 & 0.94 & 0.016 & 0.21 & 250 & 0.723827 \\
\hline & $\mathrm{P}$ & $50-58$ & 0.274 & 5.15 & 302 & 7.92 & 16.6 & 5.88 & 2.30 & 5.19 & 0.014 & 0.31 & 369 & 0.725281 \\
\hline Sag1-DP & $\mathrm{P}$ (deep) & $200-300$ & 0.257 & 12.7 & 435 & 9.86 & 18.1 & 4.10 & 6.02 & 3.51 & 0.041 & 0.70 & 309 & 0.726095 \\
\hline \multirow{6}{*}{$\operatorname{AR}(n=3)$} & $\mathrm{O}$ & $0-11$ & 0.348 & 70.0 & 136 & 4.30 & 16.0 & 20.9 & 58.4 & 19.8 & 0.069 & 4.36 & 1020 & $0.708900 *$ \\
\hline & A & $11-27$ & 0.394 & 14.4 & 385 & 3.26 & 8.52 & 19.7 & 20.4 & 9.08 & 0.036 & 1.68 & 397 & $0.713011^{* *}$ \\
\hline & B1 & $27-50$ & 0.450 & 14.7 & 535 & 7.76 & 17.6 & 11.3 & 8.80 & 5.44 & 0.027 & 0.84 & 538 & 0.711376 \\
\hline & B2 & $45-75$ & 0.359 & 18.9 & 502 & 6.69 & 16.6 & 9.57 & 3.99 & 2.67 & 0.027 & 1.14 & 708 & 0.713535 \\
\hline & $\mathrm{P} 1$ & $70-80$ & 0.402 & 20.1 & 508 & 8.50 & 17.8 & 8.94 & 4.06 & 3.25 & 0.028 & 1.13 & 723 & 0.711658 \\
\hline & $\mathrm{P} 2$ & $85-93$ & 0.386 & 9.08 & 491 & 7.97 & 17.2 & 9.73 & 4.64 & 1.91 & 0.022 & 0.53 & 407 & $0.716740^{*}$ \\
\hline \multirow[t]{7}{*}{ GM $(n=2)$} & Oe & $0-13$ & 0.457 & 48.1 & 68.9 & 5.85 & 15.9 & 21.7 & 38.7 & 16.2 & 0.062 & 3.02 & 781 & $0.712801 * *$ \\
\hline & $\mathrm{B} 1$ & $13-35$ & 0.297 & 5.67 & 234 & 6.72 & 17.5 & 7.95 & 9.44 & 5.31 & 0.019 & 0.32 & 300 & $0.713987^{*}$ \\
\hline & $\mathrm{B} 2$ & $38-44$ & 0.360 & 5.84 & 460 & 8.00 & 17.1 & 9.02 & 8.52 & 5.91 & 0.021 & 0.34 & 283 & $0.714585^{*}$ \\
\hline & IIOa & $44-55$ & 0.438 & 60.6 & 204 & 4.96 & 16.4 & 15.9 & 48.5 & 16.1 & 0.066 & 3.68 & 919 & $0.713590 *$ \\
\hline & P1 & $50-54$ & 0.428 & 25.2 & 189 & 8.38 & 17.7 & 11.5 & 36.1 & 19.2 & 0.042 & 1.43 & 604 & $0.713149^{*}$ \\
\hline & IIB & $57-90$ & 0.463 & 58.4 & 209 & 14.4 & 18.1 & 8.30 & 46.1 & 12.6 & 0.066 & 3.22 & 890 & $0.712507 * *$ \\
\hline & $\mathrm{P} 2$ & $100-110$ & 0.340 & 31.7 & 211 & 14.0 & 18.8 & 5.23 & 23.2 & 4.57 & 0.046 & 1.69 & 684 & $0.714035^{* *}$ \\
\hline
\end{tabular}

Errors for elemental data and ${ }^{87} \mathrm{Sr} /{ }^{86} \mathrm{Sr}$ as reported in text, with the following exceptions:

* Error $(2 \sigma)$ greater than 0.000030 but less than 0.000075 .

** Error $(2 \sigma)$ greater than 0.000075 but less than 0.000211 .

*** $n=1$ for all ${ }^{87} \mathrm{Sr} /{ }^{86} \mathrm{Sr}$ data.

$* * * * n=2$ for It $1 \mathrm{O}$ horizon.

$1 \mu \mathrm{mol} \mathrm{L}{ }^{-1}$ in two streams that were lake outlets, but other $\mathrm{Si}$ concentrations ranged from 10 to $87 \mu \mathrm{mol} \mathrm{L}{ }^{-1} \cdot{ }^{87} \mathrm{Sr} /{ }^{86} \mathrm{Sr}$ ranged from 0.71023 to 0.71881 in streams draining glacial deposit surfaces and from 0.70847 to 0.71989 in streams draining bedrock surfaces.

\section{Discussion}

\section{ANALYSIS OF SOIL PARENT MATERIAL VARIABILITY}

Before we can evaluate whether geochemical patterns are dependent on varying degrees of weathering on geomorphic surfaces of different ages, we must ascertain the degree of variability of the composition of the glacial till soil parent among the surfaces. Figure 2 shows a comparison of the ${ }^{87} \mathrm{Sr} /{ }^{86} \mathrm{Sr}$ of the hot acid digestible fractions of the soil profiles on each surface. Because easily weathered material has already been removed from this fraction by previous steps in the sequential digest procedure, we make the assumption that the chemistry of this fraction reflects minerals that would only be affected by intense weathering, making them unlikely to have been significantly affected yet by weathering in this arctic region. Therefore, the ${ }^{87} \mathrm{Sr} /{ }^{86} \mathrm{Sr}$ and elemental ratios of this fraction should be very similar for soils with similar parent material, regardless of their age. We used 
TABLE 5

Total soil digest chemistry (per gram dry soil). Surface and horizon names are defined in the text.

\begin{tabular}{|c|c|c|c|c|c|c|c|c|c|c|c|c|c|c|}
\hline Surface & Horizon & $\begin{array}{l}\text { Depth } \\
(\mathrm{cm})\end{array}$ & $\begin{array}{c}\mathbf{B a} \\
(\mu \mathrm{mol} / \mathrm{g})\end{array}$ & $\begin{array}{c}\text { Ca } \\
(\mu \mathrm{mol} / \mathrm{g})\end{array}$ & $\begin{array}{c}\mathbf{F e} \\
(\mu \mathrm{mol} / \mathrm{g})\end{array}$ & $\begin{array}{c}\mathbf{K} \\
(\mu \mathrm{mol} / \mathrm{g})\end{array}$ & $\begin{array}{c}\mathbf{N a} \\
(\mu \mathrm{mol} / \mathrm{g})\end{array}$ & $\begin{array}{c}\mathbf{P} \\
(\mu \mathrm{mol} / \mathrm{g})\end{array}$ & $\begin{array}{c}\mathbf{S i} \\
(\mu \mathrm{mol} / \mathrm{g})\end{array}$ & $\begin{array}{c}\mathbf{S r} \\
(\mu \mathrm{mol} / \mathrm{g})\end{array}$ & $\begin{array}{c}\mathbf{C a} / \mathbf{N a} \\
\text { (molar) }\end{array}$ & $\begin{array}{c}\mathbf{C a} / \mathrm{Sr} \\
\text { (molar) }\end{array}$ & $\begin{array}{l}\text { Fraction } \\
(<2 \mathrm{~mm})\end{array}$ & $\begin{array}{l}\text { LOI } \\
(\%)\end{array}$ \\
\hline \multirow[t]{5}{*}{ It3 $(n=4)$} & $\mathrm{O}$ & $0-7$ & - & - & - & - & - & - & - & - & - & - & - & - \\
\hline & A & $7-19$ & 3.01 & 20.5 & 547 & 282 & 128 & 12.73 & 13600 & 0.583 & 0.017 & 35.2 & $76 \%$ & $5 \%$ \\
\hline & B1 & $19-39$ & 3.50 & 19.9 & 685 & 325 & 129 & 14.68 & 14100 & 0.624 & 0.018 & 31.9 & $76 \%$ & $7 \%$ \\
\hline & B2 & $39-60$ & 3.51 & 19.7 & 644 & 327 & 122 & 16.77 & 12800 & 0.585 & 0.019 & 33.7 & $80 \%$ & $8 \%$ \\
\hline & $\mathrm{P}$ & $62-71$ & 4.66 & 35.1 & 730 & 383 & 128 & 23.84 & 13200 & 0.643 & 0.023 & 54.6 & $63 \%$ & - \\
\hline \multirow[t]{5}{*}{ It2 $(n=3)$} & $\mathrm{O}$ & $0-16$ & - & - & - & - & - & - & - & - & - & - & - & - \\
\hline & A & $18-29$ & 4.77 & 124 & 504 & 213 & 151 & 22.23 & 13900 & 0.577 & 0.023 & 214 & $65 \%$ & $12 \%$ \\
\hline & B1 & $29-46$ & 5.32 & 113 & 565 & 271 & 155 & 19.90 & 14000 & 0.591 & 0.023 & 192 & $52 \%$ & $20 \%$ \\
\hline & B2 & $46-62$ & 5.73 & 202 & 606 & 265 & 141 & 23.04 & 12400 & 0.626 & 0.024 & 323 & $59 \%$ & $8 \%$ \\
\hline & $\mathrm{P}$ & $66-73$ & 6.67 & 330 & 831 & 335 & 168 & 27.77 & 11800 & 0.759 & 0.021 & 434 & $82 \%$ & $7 \%$ \\
\hline \multirow[t]{6}{*}{ It1 $(n=3)$} & $\mathrm{O}^{*}$ & $0-7$ & - & - & - & - & - & - & - & - & - & - & - & - \\
\hline & A & $8-23$ & 2.65 & 18.2 & 541 & 251 & 148 & 12.80 & 15300 & 0.508 & 0.019 & 35.8 & $49 \%$ & $5 \%$ \\
\hline & $\mathrm{B} 1$ & $23-40$ & 2.85 & 18.7 & 484 & 262 & 118 & 15.31 & 15400 & 0.526 & 0.019 & 35.5 & $57 \%$ & $8 \%$ \\
\hline & B2 & $35-49$ & 3.30 & 20.4 & 638 & 286 & 108 & 17.46 & 15100 & 0.562 & 0.020 & 36.3 & $54 \%$ & - \\
\hline & $\mathrm{P} 1$ & $49-60$ & 3.24 & 19.9 & 586 & 304 & 112 & 15.13 & 17200 & 0.555 & 0.020 & 35.9 & $53 \%$ & - \\
\hline & $\mathrm{P} 2$ & $62-69$ & 3.45 & 108 & 653 & 305 & 117 & 16.98 & 15100 & 0.635 & 0.020 & 170 & $71 \%$ & $10 \%$ \\
\hline \multirow[t]{6}{*}{ Sag2 $(n=3)$} & $\mathrm{O}$ & $0-8$ & - & - & - & - & - & - & - & - & - & - & - & - \\
\hline & A & $8-25$ & 2.66 & 19.4 & 466 & 197 & 101 & 14.25 & 15600 & 0.466 & 0.021 & 41.6 & $66 \%$ & $7 \%$ \\
\hline & B1 & $25-42$ & 4.37 & 30.1 & 705 & 315 & 124 & 20.55 & 13500 & 0.517 & 0.024 & 58.3 & $79 \%$ & $23 \%$ \\
\hline & B2 & $38-55$ & 2.61 & 23.8 & 343 & 195 & 115 & 8.60 & 16100 & 0.505 & 0.019 & 47.1 & $69 \%$ & - \\
\hline & $\mathrm{P} 1$ & $55-69$ & 2.55 & 22.5 & 514 & 179 & 93.5 & 14.53 & 15200 & 0.454 & 0.021 & 49.4 & $61 \%$ & - \\
\hline & $\mathrm{P} 2$ & $71-75$ & 3.82 & 37.5 & 743 & 265 & 112 & 17.95 & 13400 & 0.432 & 0.026 & 86.8 & $77 \%$ & $22 \%$ \\
\hline \multirow[t]{5}{*}{ Sag1 $(n=2)$} & $\mathrm{O}$ & $0-13$ & - & - & - & - & - & - & - & - & - & - & - & - \\
\hline & A & $13-22$ & 3.67 & 29.3 & 565 & 236 & 137 & 17.1 & 14000 & 0.560 & 0.023 & 52.3 & $69 \%$ & $15 \%$ \\
\hline & B1 & $22-40$ & 3.55 & 26.6 & 519 & 229 & 157 & 12.36 & 14000 & 0.548 & 0.023 & 48.5 & $65 \%$ & $7 \%$ \\
\hline & B2 & $35-50$ & 4.08 & 26.8 & 586 & 264 & 167 & 13.29 & 14400 & 0.576 & 0.024 & 46.5 & $58 \%$ & - \\
\hline & $\mathrm{P}$ & $50-58$ & 3.77 & 25.9 & 525 & 243 & 140 & 13.79 & 14200 & 0.570 & 0.024 & 45.4 & $61 \%$ & $7 \%$ \\
\hline Sag1-DP & P (deep) & $200-300$ & 7.13 & 255 & 896 & 675 & 153 & 29.46 & 13400 & 1.14 & 0.019 & 224 & $88 \%$ & - \\
\hline \multirow[t]{6}{*}{$\operatorname{AR}(n=3)$} & $\mathrm{O}$ & $0-11$ & - & - & - & - & - & - & - & - & - & - & - & - \\
\hline & A & $11-27$ & 4.13 & 138 & 581 & 200 & 181 & 30.1 & 11900 & 0.589 & 0.021 & 234 & $46 \%$ & - \\
\hline & B1 & $27-50$ & 5.34 & 270 & 631 & 267 & 239 & 31.6 & 13500 & 0.797 & 0.019 & 339 & $40 \%$ & - \\
\hline & B2 & $45-75$ & 4.58 & 428 & 529 & 255 & 199 & 26.89 & 11900 & 0.858 & 0.017 & 498 & $30 \%$ & - \\
\hline & $\mathrm{P} 1$ & $70-80$ & 5.45 & 483 & 593 & 268 & 235 & 32.41 & 13000 & 0.915 & 0.020 & 528 & $41 \%$ & - \\
\hline & $\mathrm{P} 2$ & $85-93$ & 5.25 & 612 & 596 & 277 & 227 & 48.97 & 13700 & 1.27 & 0.019 & 480 & $37 \%$ & - \\
\hline \multirow[t]{7}{*}{ GM $(n=2)$} & Oe & $0-13$ & - & - & - & - & - & - & - & - & - & - & - & - \\
\hline & $\mathrm{B} 1$ & $13-35$ & 4.47 & 67.6 & 475 & 259 & 222 & 20.99 & 13200 & 0.685 & 0.023 & 98.7 & $47 \%$ & - \\
\hline & B2 & $38-44$ & 4.37 & 75.7 & 845 & 209 & 183 & 20.33 & 12600 & 0.682 & 0.024 & 111 & $76 \%$ & - \\
\hline & IIOa & $44-55$ & 3.16 & 289 & 379 & 128 & 95.5 & 28.8 & 5860 & 0.614 & 0.032 & 471 & $69 \%$ & - \\
\hline & $\mathrm{P} 1$ & $50-54$ & 3.65 & 219 & 336 & 164 & 126 & 24.9 & 8240 & 0.659 & 0.028 & 333 & $57 \%$ & - \\
\hline & IIB & $57-90$ & 4.60 & 593 & 396 & 249 & 203 & 25.30 & 10600 & 0.982 & 0.020 & 604 & $79 \%$ & - \\
\hline & $\mathrm{P} 2$ & $100-110$ & 4.82 & 1320 & 452 & 283 & 208 & 27.27 & 11400 & 1.63 & 0.011 & 814 & $57 \%$ & - \\
\hline
\end{tabular}

Errors for elemental data as reported in text. LOI $=$ loss on ignition.

${ }^{87} \mathrm{Sr} /{ }^{86} \mathrm{Sr}$ rather than elemental ratios as an indicator of differences in parent material because different parent materials of the same type (e.g. different sandstone units) may have very similar elemental ratios due to similar mineralogical compositions, but because of their unique protoliths, they are more likely to have detectable differences in ${ }^{87} \mathrm{Sr} /{ }^{86} \mathrm{Sr}$. We examined the ${ }^{87} \mathrm{Sr} /{ }^{86} \mathrm{Sr}$ of the hot acid digestible fraction with respect to depth, to account for any minor effects of weathering or addition of new material (e.g. eolian loess) to the soil profile.

Overall the composition of the parent material has low variability but showed some grouping among the different surfaces. The GM and AR profiles have significantly lower mean ${ }^{87} \mathrm{Sr} /{ }^{86} \mathrm{Sr}$ than the other surfaces (Fig. $2 ; p<0.05$ ). This suggests that the AR and GM soils are derived from different parent material than the other surfaces. Differences in parent material may be explained by the incorporation of various rock units in different proportions based on different surface expressions of these units at the time of the glacial advances. Additionally, rock units (the $\mathrm{Kn}$ and three minor units not analyzed here) that outcrop north of the Sag1 surface may have been incorporated into the GM and AR parent till. It is also possible that long-term eolian loess deposition has contributed to these differences in soil composition. The ${ }^{87} \mathrm{Sr} /{ }^{86} \mathrm{Sr}$ of the hot acid digestible fraction of the It1, It2, It3, Sag1, and Sag2 soil profiles is similar, suggesting that these surfaces have similar parent material, although the ${ }^{87} \mathrm{Sr} /{ }^{86} \mathrm{Sr}$ of the upper part of the It3 profile is slightly higher than that of the other surfaces. This is consistent with the suggestion by Hamilton (2003) that the It3 surface was derived from slightly different parent material than the It 1 and It2 surfaces, based on surficial geomorphology. For the purposes of further analyses 
TABLE 6

Water chemistry for streams on different glacial deposit and bedrock surfaces. Surface names are defined in the text.

\begin{tabular}{|c|c|c|c|c|c|c|c|c|c|c|c|c|c|c|}
\hline Surface & Stream name & $\begin{array}{c}\text { Date } \\
\text { sampled }\end{array}$ & $\begin{array}{c}\text { Ba } \\
(\mu \mathrm{mol} / \mathrm{L})\end{array}$ & $\begin{array}{c}\text { Ca } \\
(\mu \mathrm{mol} / \mathrm{L})\end{array}$ & $\begin{array}{c}\mathbf{F e} \\
(\mu \mathrm{mol} / \mathrm{L})\end{array}$ & $\begin{array}{c}\mathbf{K} \\
(\mu \mathrm{mol} / \mathrm{L})\end{array}$ & $\begin{array}{c}\text { Mg } \\
(\mu \mathrm{mol} / \mathrm{L})\end{array}$ & $\begin{array}{c}\mathbf{N a} \\
(\mu \mathrm{mol} / \mathrm{L})\end{array}$ & $\begin{array}{c}\mathbf{S} \\
(\mu \mathrm{mol} / \mathrm{L})\end{array}$ & $\begin{array}{c}\mathbf{S i} \\
(\mu \mathrm{mol} / \mathrm{L})\end{array}$ & $\begin{array}{c}\mathbf{S r} \\
(\mu \mathrm{mol} / \mathrm{L})\end{array}$ & $\begin{array}{r}\mathbf{C a} / \mathbf{N a} \\
\text { (molar) }\end{array}$ & $\begin{array}{c}\mathbf{C a} / \mathbf{S r} \\
\text { (molar) }\end{array}$ & ${ }^{87} \mathrm{Sr} /{ }^{86} \mathrm{Sr}$ \\
\hline \multirow[t]{2}{*}{ It3 } & (1) It 3 stream & $7 / 29 / 02$ & 0.08 & 551.3 & 3.88 & 2.3 & 104.9 & 36 & 20.8 & 67.6 & 0.740 & 15 & 745 & $0.710391 *$ \\
\hline & (2) Island Lake outlet & t $8 / 2 / 02$ & 0.13 & 522.0 & $<0.08$ & 10.3 & 183.5 & 77 & 20.7 & 85.9 & 0.736 & 6.8 & 709 & 0.710234 \\
\hline \multirow[t]{4}{*}{ It2 (outwash) } & (3) I minus outlet & $6 / 27 / 02$ & 0.10 & 219.6 & 1.03 & 8.3 & 61.5 & 16 & 20.8 & 78.2 & 0.760 & 14 & 289 & 0.713132 \\
\hline & (4) I minus inlet & $6 / 27 / 02$ & 0.07 & 136.3 & 2.26 & 4.8 & 37.9 & 13 & 20.8 & 78.8 & 0.766 & 11 & 178 & 0.712971 \\
\hline & $\begin{array}{l}\text { (5) TW stream } \\
\text { (lower) }\end{array}$ & $7 / 21 / 02$ & 0.10 & 269.2 & 0.96 & 1.0 & 57.6 & 7 & 20.8 & 77.9 & 0.761 & 40 & 354 & 0.713314 \\
\hline & $\begin{array}{l}\text { (6) Milky Way } \\
\text { (upper) }\end{array}$ & $8 / 10 / 02$ & 0.12 & 255.4 & 1.56 & 2.3 & 62.7 & 27 & 18.4 & 40.3 & 0.205 & 9.4 & 1244 & $0.712085^{*}$ \\
\hline \multirow[t]{4}{*}{ It2 (till) } & (7) N1 inlet & $7 / 11 / 02$ & 0.09 & 543.5 & 0.64 & 1.2 & 117.5 & 13 & 20.8 & 80.3 & 0.752 & 42 & 722 & $0.710600 *$ \\
\hline & (8) Il outlet & $8 / 7 / 02$ & 0.03 & 123.1 & 1.34 & 6.4 & 36.5 & 14 & 8.1 & 0.9 & 0.102 & 8.8 & 1204 & $0.715188 * *$ \\
\hline & (9) I4 outlet & $8 / 7 / 02$ & 0.05 & 104.9 & 2.65 & 5.0 & 34.7 & 13 & 8.5 & $<0.4$ & 0.096 & 7.8 & 1093 & 0.715593 \\
\hline & (10) I2 outlet & $8 / 7 / 02$ & 0.07 & 129.7 & 4.61 & 6.7 & 50.5 & 14 & 6.8 & 10.4 & 0.131 & 9.4 & 988 & $0.716473^{*}$ \\
\hline \multirow[t]{3}{*}{ It1 } & (11) I6 HW inlet & $8 / 8 / 02$ & 0.08 & 594.3 & 9.20 & 1.0 & 97.9 & 20 & 6.4 & 34.6 & 0.404 & 30 & 1471 & 0.711281 \\
\hline & (12) I8 Headwater & $6 / 26 / 02$ & 0.06 & 168.1 & 2.32 & 2.8 & 52.7 & 13 & 20.8 & 78.8 & 0.764 & 13 & 220 & $0.713464 *$ \\
\hline & (13) It 1 stream & $7 / 15 / 02$ & 0.25 & 440.6 & $<0.08$ & 2.5 & 73.4 & 19 & 20.8 & 76.2 & 0.746 & 23 & 590 & 0.710995 \\
\hline \multirow[t]{2}{*}{ Sag2 } & (14) E5 inlet S & $7 / 30 / 02$ & 0.07 & 44.2 & 6.37 & 0.7 & 20.2 & 11 & 3.1 & 37.2 & 0.046 & 4.0 & 958 & 0.716328 \\
\hline & (15) E5 inlet W & $8 / 13 / 02$ & 0.09 & 19.7 & 2.02 & 0.3 & 12.2 & 13 & 8.5 & 43.6 & 0.025 & 1.6 & 796 & 0.718806 \\
\hline \multirow[t]{2}{*}{ Sag1 } & (16) Imnavait (weir) & $\begin{array}{l}8 / 1 / \\
2002\end{array}$ & 0.08 & 28.1 & 13.55 & 0.6 & 14.2 & 4 & 1.2 & 20.2 & 0.036 & 7 & 777 & 0.716905 \\
\hline & (17) Toolik R. trib. & $8 / 16 / 02$ & 0.06 & 11.7 & 8.36 & 0.5 & 10.9 & 4 & 2.6 & 29.0 & 0.018 & 3 & 654 & 0.717341 \\
\hline $\mathbf{A R}$ & $\begin{array}{l}\text { (18) Anaktuvuk } \\
\text { stream }\end{array}$ & $8 / 15 / 03$ & 0.08 & 78.2 & 4.55 & 0.6 & 18.3 & 12 & 2.3 & 47.1 & 0.079 & 6.7 & 990 & 0.712417 \\
\hline GM & (19) Gunsight stream & $8 / 16 / 03$ & 0.07 & 165.8 & 5.62 & $<0.3$ & 44.2 & 9 & 3.5 & 58.7 & 0.148 & 20 & 1120 & 0.711356 \\
\hline \multirow{2}{*}{$\begin{array}{l}\text { Multiple } \\
\text { surfaces }\end{array}$} & (20) Kuparuk R. & $8 / 7 / 02$ & 0.28 & 199.1 & 0.37 & 6.6 & 71.2 & 78 & 20.8 & 65.3 & 0.745 & 2.6 & 267 & $0.707878 * *$ \\
\hline & (21) Sagavanirktok R. & $.7 / 31 / 02$ & 0.22 & 924.3 & $<0.08$ & 8.9 & 282.5 & 80 & 20.7 & 76.8 & 0.635 & 12 & 1456 & 0.710453 \\
\hline \multirow{3}{*}{$\begin{array}{r}\text { Carbonate } \\
\text { bedrock }\end{array}$} & (22) Atigun R. trib. & $7 / 29 / 02$ & 0.26 & 1559.1 & $<0.08$ & 13.3 & 287.4 & 97 & 20.6 & 80.2 & 0.678 & 16 & 2301 & $0.710550^{*}$ \\
\hline & (23) Kayak Creek & $8 / 7 / 02$ & 0.13 & 1605.7 & $<0.08$ & 7.5 & 166.3 & 20 & 20.8 & 80.9 & 0.569 & 79 & 2821 & 0.708469 \\
\hline & (24) Aufeis stream & $7 / 31 / 02$ & 0.35 & 1226.5 & $<0.08$ & 5.4 & 272.0 & 21 & 20.8 & 71.0 & 0.707 & 58 & 1736 & 0.708802 \\
\hline \multirow{5}{*}{$\begin{array}{l}\text { Mixed } \\
\text { bedrock }\end{array}$} & (25) Trevor Creek & $8 / 7 / 02$ & 0.30 & 706.7 & $<0.08$ & 10.5 & 264.2 & 94 & 20.7 & 83.6 & 0.704 & 7.5 & 1003 & $0.714855^{*}$ \\
\hline & (26) Atigun R. & $7 / 29 / 02$ & 0.18 & 691.1 & $<0.08$ & 12.2 & 324.6 & 122 & 20.7 & 80.8 & 0.697 & 5.67 & 991 & 0.714458 \\
\hline & (27) Bad Idea stream & $8 / 18 / 03$ & 0.18 & 866.5 & $<0.08$ & 14.8 & 167.5 & 169 & 397.0 & 34.7 & 1.495 & 5.14 & 580 & 0.711382 \\
\hline & (28) Holden Creek & $8 / 18 / 03$ & 0.09 & 569.4 & 0.14 & 8.6 & 102.4 & 88 & 122.5 & 21.2 & 1.212 & 6.4 & 470 & 0.709772 \\
\hline & $\begin{array}{l}\text { (29) Roche } \\
\text { Moutonee Cr. }\end{array}$ & $8 / 18 / 03$ & 0.12 & 372.6 & 0.16 & 9.9 & 110.1 & 56 & 83.0 & 24.0 & 0.465 & 6.7 & 802 & 0.713843 \\
\hline \multirow[t]{2}{*}{$\begin{array}{l}\text { Silicate } \\
\text { bedrock }\end{array}$} & $\begin{array}{l}\text { (30) Atigun R. } \\
\text { headwater }\end{array}$ & $8 / 7 / 02$ & 0.07 & 570.4 & $<0.08$ & 12.2 & 395.8 & 271 & 20.7 & 77.5 & 0.686 & 2.11 & 832 & $0.719655^{*}$ \\
\hline & $\begin{array}{l}\text { (31) Underwillow } \\
\text { stream }\end{array}$ & $8 / 18 / 03$ & 0.27 & 254.5 & 0.30 & 22.7 & 194.9 & 296 & 318.6 & 31.6 & 0.502 & .86 & 507 & 0.719893 \\
\hline \multirow[t]{2}{*}{ Precipitation } & field station precip. 1 & $8 / 5 / 02$ & $<0.01$ & 102.2 & $<0.08$ & 83.5 & 4.4 & 218 & 20.8 & 92.3 & 0.770 & 0.47 & 133 & $0.709485^{* *}$ \\
\hline & field station precip. 2 & $8 / 6 / 02$ & 0.03 & 84.9 & $<0.08$ & 19.0 & 2.1 & 57 & 20.8 & 92.0 & 0.772 & 1.5 & 110 & $0.708781^{*}$ \\
\hline
\end{tabular}

Errors for elemental data and ${ }^{87} \mathrm{Sr} /{ }^{86} \mathrm{Sr}$ as reported in text, with the following exceptions:

* Error $(2 \sigma)$ greater than 0.000030 but less than 0.000051 .

** error $(2 \sigma)$ greater than 0.000051 but less than 0.000165 .

with respect to surface age, we will consider only the It1, It2, Sag1, and Sag2 to be derived from similar parent material.

Besides these overall differences among the profiles from each surface, each individual profile shows a pattern of increasing hot acid digestible fraction ${ }^{87} \mathrm{Sr} /{ }^{86} \mathrm{Sr}$ with increasing depth within the active layer (down to approximately $50 \mathrm{~cm}$; Fig. 2). The most likely explanation for this is eolian deposition of a weatheringresistant mineral, with lower ${ }^{87} \mathrm{Sr} /{ }^{86} \mathrm{Sr}$ than the soil parent material, which is mixed down through the active layer by cryoturbation or water movement. Such dust could be derived from the Dke sandstone or resistant minerals in the PMl (Table 1), or from sediments outside the study region. This dust may also have a carbonate component that contributes to the exchangeable and cold acid digestible fractions. However, the effects of this input as shown by increasing ${ }^{87} \mathrm{Sr} /{ }^{86} \mathrm{Sr}$ with depth seem to be of similar magnitude in the hot acid digestible fraction, so we can assume that age comparisons between these surfaces based on the other fractions are not substantially affected. An alternate explanation for the pattern of increasing ${ }^{87} \mathrm{Sr} /{ }^{86} \mathrm{Sr}$ with depth in the active layer could be removal by weathering of a resistant mineral with relatively high ${ }^{87} \mathrm{Sr} /{ }^{86} \mathrm{Sr}$ (such as biotite) in the upper part of the active layer, but this seems unlikely due to the low weathering intensity of this environment as evidenced by the relatively slow carbonate weathering described in the next section. 


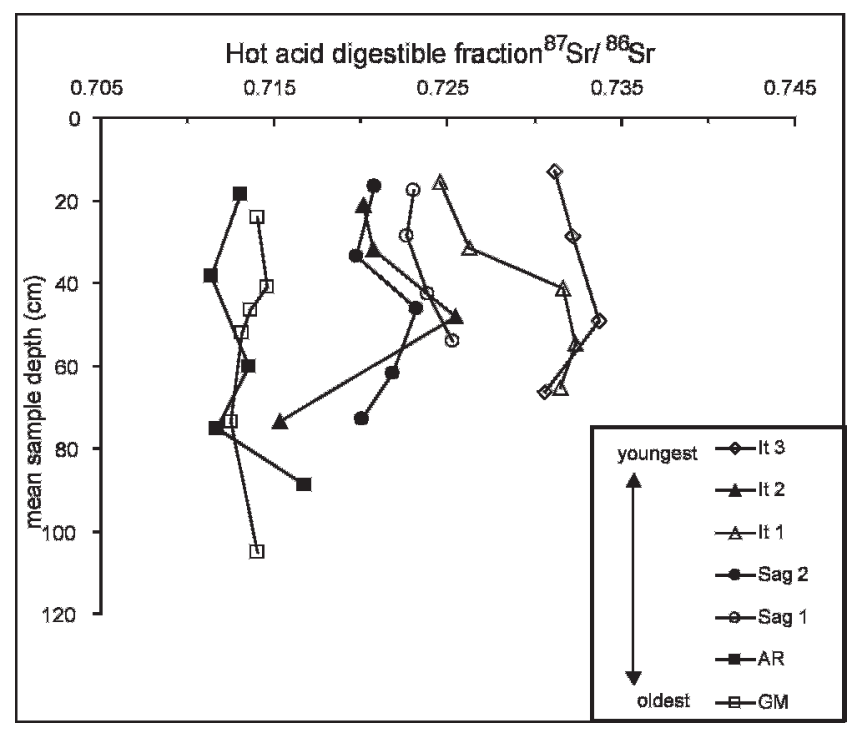

FIGURE 2. Hot acid digestible fraction ${ }^{87} \mathrm{Sr} /{ }^{86} \mathrm{Sr}$ of soil horizon samples vs. mean horizon depth. ${ }^{87} \mathrm{Sr} /{ }^{86} \mathrm{Sr}$ analytical errors are reported in Table 4.

\section{CARBONATE WEATHERING AND ITS RELATIONSHIP TO SURFACE AGE}

Calcite depletion via weathering in the active layer is indicated on every surface, by decreasing ${ }^{87} \mathrm{Sr} /{ }^{86} \mathrm{Sr}$ and increasing $\mathrm{Ca} / \mathrm{Na}$ and $\mathrm{Ca} / \mathrm{Sr}$ with depth (Fig. 3), and by greater $\mathrm{Ca}$ concentrations in permafrost than in active layer mineral soil as described in the results section. These geochemical trends suggest increasing carbonate mineral concentration in the soil with depth, as frequency of thaw and hence weathering intensity decreases. These results may explain the findings of Everett et al. (1989), who monitored the seasonal geochemistry of the Imnavait stream on the Sag1 surface and found that streamwater $\mathrm{Ca}$ concentrations were greatest in the late summer, corresponding to deep seasonal thaw.

When considering only the surfaces with similar parent material (It1, It2, Sag1, and Sag2), some patterns related to surface age become apparent. The mean ${ }^{87} \mathrm{Sr} /{ }^{86} \mathrm{Sr}$ values of the active-layer cold acid digestible fraction are lowest on the youngest surface (It2) and highest on the oldest (Sag1), with overlapping values for the two other surfaces (Fig. 3c). The decrease in mean cold acid digestible fraction ${ }^{87} \mathrm{Sr} /{ }^{86} \mathrm{Sr}$ between the permafrost and the " $A$ " horizon is least on the youngest surface. The youngest surface also has the highest exchangeable, acid digestible, and total $\mathrm{Ca}$ concentrations and $\mathrm{Ca} / \mathrm{Sr}$ (Tables 2, 3, and 5). These data suggest that weathering has progressively depleted the calcite in the active layer over time on these surfaces. Furthermore, mean cold acid digestible fraction ${ }^{87} \mathrm{Sr} /{ }^{86} \mathrm{Sr}$ values for shallow permafrost ("P" samples from within soil pits, excluding the Sag1 deep permafrost sample) increase with surface age among the four surfaces derived from similar parent material (Fig. 3c, Table 3). This trend suggests that the currently frozen soil has been episodically thawed during past intervals of warmer climate and has undergone some weathering or was mixed with weathered material via cryoturbation during these intervals.

Both stream and soil geochemistry suggest that differences in carbonate weathering drive not only depth-related geochemical differences within individual soil profiles, but also age-related differences in whole watershed geochemistry. Simple chemical mixing lines drawn between end-members formed by the cold acid digestible fractions of the PMl limestone (high $\mathrm{Ca} / \mathrm{Sr}$, low (a)
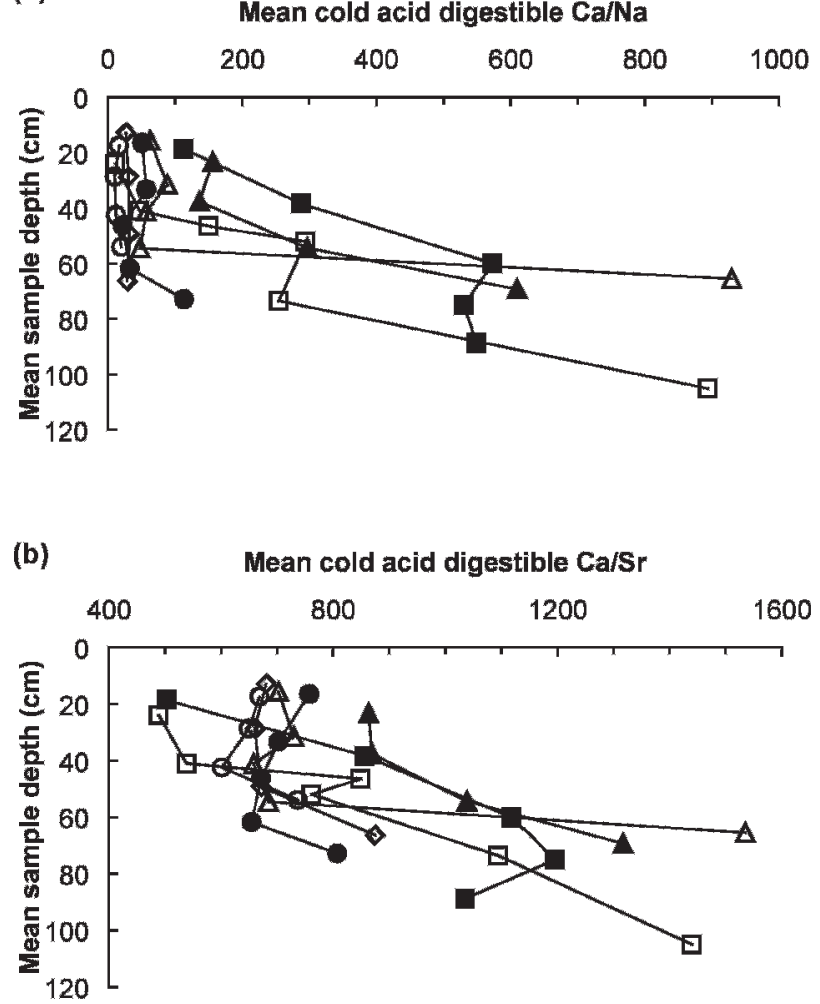

(c)

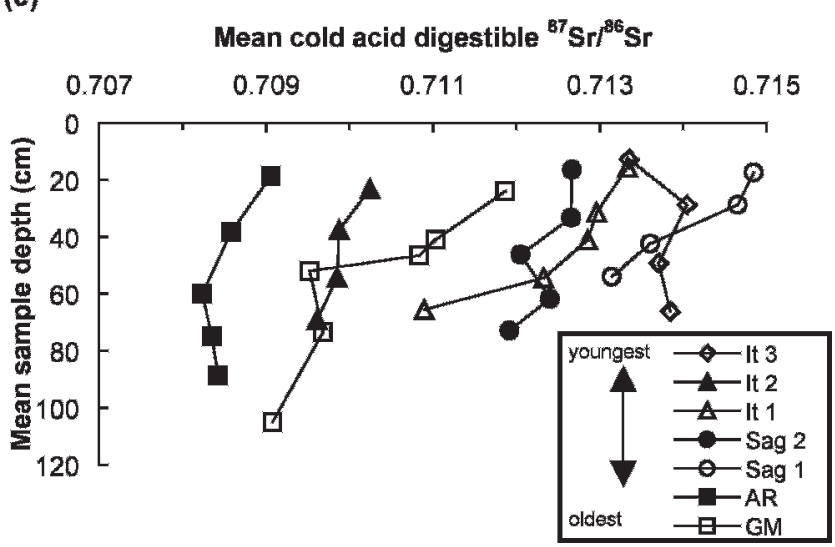

FIGURE 3. Cold acid digestible (a) $\mathrm{Ca} / \mathrm{Na}$, (b) $\mathrm{Ca} / \mathrm{Sr}$, and (c) ${ }^{87} \mathrm{Sr} /{ }^{86} \mathrm{Sr}$ of soil horizon samples vs. mean sample depth. Elemental ratios are molar. Analytical errors are reported in the text.

${ }^{87} \mathrm{Sr} /{ }^{86} \mathrm{Sr}$ ), Dke sandstone (low $\mathrm{Ca} / \mathrm{Sr}$, high ${ }^{87} \mathrm{Sr} /{ }^{86} \mathrm{Sr}$ ), and $\mathrm{Kf}$ sandstone (low $\mathrm{Ca} / \mathrm{Sr}$, low ${ }^{87} \mathrm{Sr} /{ }^{86} \mathrm{Sr}$ ) represent the expected composition of waters containing easily weathered solutes derived from these units based on varying proportional contributions from each unit by mass (Fig. 4). These mixing lines define a range containing 7 out of 15 streams draining watersheds developed on different glacial till surfaces. Streams draining PMl-based watersheds have a lower mean $\mathrm{Ca} / \mathrm{Sr}$ and very slightly higher mean ${ }^{87} \mathrm{Sr} /{ }^{86} \mathrm{Sr}$ than the cold acid digestible fraction of PMl samples (Fig. 4, hatched line end-members). This may be explained by a greater influence of precipitation on these watersheds, or a fraction of the PMl which is more soluble under natural conditions and differs chemically from the fraction digestible with stronger acid in the laboratory, or both. The slightly higher $\mathrm{Ca} / \mathrm{Sr}$ and ${ }^{87} \mathrm{Sr} /{ }^{86} \mathrm{Sr}$ of streams draining Dke sandstone and undifferentiated Kanayut conglomerate (MDk) bedrock may be explained 


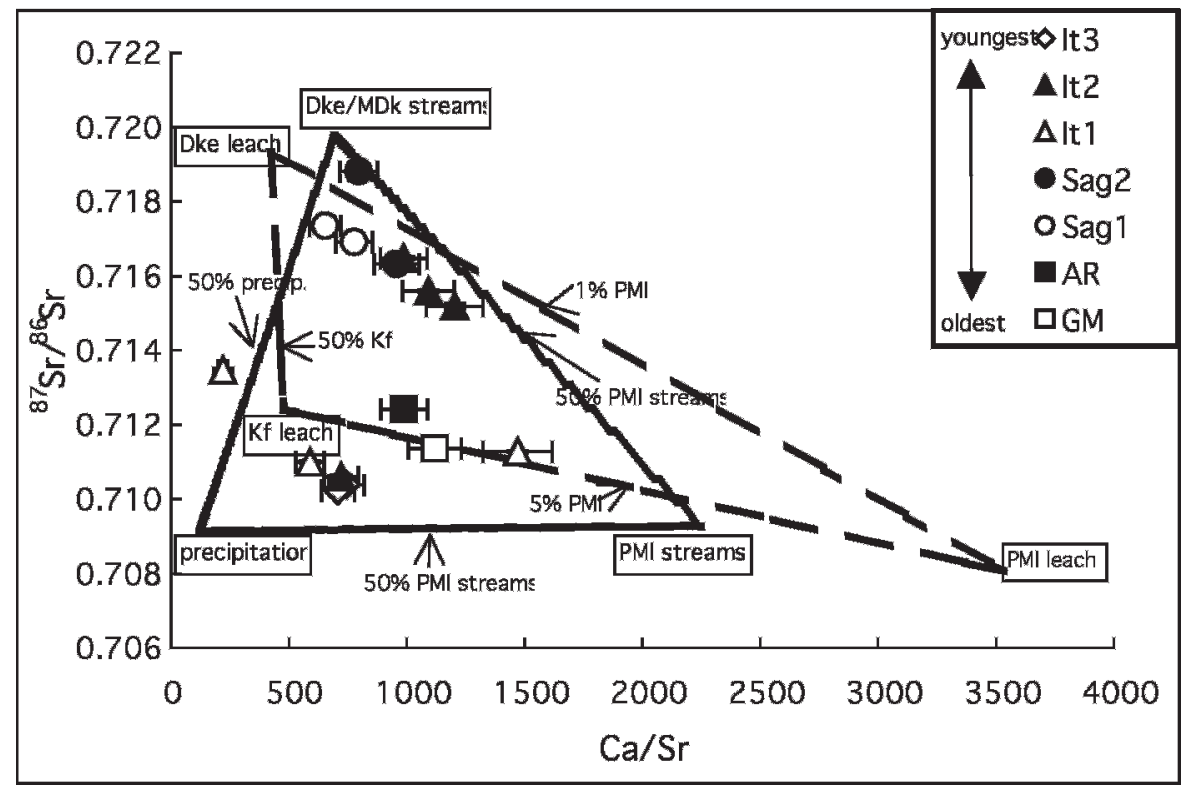

FIGURE 4. ${ }^{87} \mathrm{Sr} /{ }^{86} \mathrm{Sr}$ vs. $\mathrm{Ca} / \mathrm{Sr}$ of stream water samples from glacial deposit surfaces. Dashed lines connecting the cold acid digestible fraction values of PMI, Dke, and Kf rock units (see Table 1) represent simple chemical mixing lines for these endmembers. Hatched lines connecting the mean values for precipitation and streams draining PMI and Dke/MDk units represent simple chemical mixing lines for these end-members. $\mathrm{Ca} / \mathrm{Sr}$ is a molar ratio. Error bars represent propagated $\mathrm{Ca} / \mathrm{Sr}$ analytical errors; ${ }^{87} \mathrm{Sr} /{ }^{86} \mathrm{Sr}$ errors are smaller than the symbol size and are reported in Table 6. Rock unit abbreviations are explained in the text. by contributions from other members of the MDk, as well as the slightly higher ${ }^{87} \mathrm{Sr} /{ }^{86} \mathrm{Sr}$ of the exchangeable fraction of the Dke.

Simple chemical mixing lines drawn between the mean $\mathrm{Ca} / \mathrm{Sr}$ and ${ }^{87} \mathrm{Sr} /{ }^{86} \mathrm{Sr}$ of precipitation samples, streams draining $\mathrm{PMl}$ limestone bedrock, and streams draining Dke/MDk bedrock (Fig. 4, hatched lines) represent the expected composition of waters containing solutes derived from a combination of inputs based on the solutes measured in unit-specific streams and precipitation. All but one of the 15 glacial till surface streams sampled are contained within these mixing lines (Fig. 4). The reasons that these end-members predict water composition more accurately than the range defined by the cold acid digestible fraction of rock units are likely that (1) the effects of precipitation and other members of the MDk are incorporated, and (2) they are based on weathering in natural settings, rather than on an operationally defined laboratory cold acid digest.

Within the ranges defined by both sets of mixing lines, most streams on younger (It3, It 2 , and It 1 ) surfaces have ${ }^{87} \mathrm{Sr} /{ }^{86} \mathrm{Sr}$ ratios similar to those of streams draining limestone bedrock and the cold acid digestible fraction of limestone, but lake outlet streams on the It2 surface have higher ${ }^{87} \mathrm{Sr} /{ }^{86} \mathrm{Sr}$. The $\mathrm{Ca} / \mathrm{Sr}$ ratios of these streams vary widely, perhaps reflecting varying effects of precipitation in different watersheds or at the different times when the streams were sampled. The effects of precipitation on the ${ }^{87} \mathrm{Sr} /{ }^{86} \mathrm{Sr}$ of these streams would be nearly undetectable because the difference between the ${ }^{87} \mathrm{Sr} /{ }^{86} \mathrm{Sr}$ of precipitation and streams draining PMl watersheds is less than 0.0002. Streams on older (Sag2 and Sag1) surfaces have $\mathrm{Ca} / \mathrm{Sr}$ and ${ }^{87} \mathrm{Sr} /{ }^{86} \mathrm{Sr}$ ratios similar to those of streams draining Dke/MDk bedrock and the cold acid digestible fraction of Dke sandstone, suggesting much less carbonate influence on these surfaces than on younger surfaces. Streams on very old (AR and GM) surfaces have $\mathrm{Ca} / \mathrm{Sr}$ and ${ }^{87} \mathrm{Sr} /{ }^{86} \mathrm{Sr}$ which fall very close to the Kf-PMl mixing line, suggesting that the weathering products dissolved in these streams are primarily derived from these two end-members, or perhaps an end-member with similar chemistry such as the Kn (Table 1). The chemistry of the GM and AR streams suggests little input of the Dke/MDk end-member, consistent with the differences in parent material between these and younger surfaces discussed above.

The effects of weathering on shallow mineral soils of different ages was examined by plotting cold acid digestible $\mathrm{Ca} / \mathrm{Sr}$ vs.
${ }^{87} \mathrm{Sr} /{ }^{86} \mathrm{Sr}$ for soil "A" horizon samples from all glacial deposit surfaces along with the mixing lines from the cold acid digestible fraction rock unit end-members described above (Fig. 5). $\mathrm{Ca} / \mathrm{Sr}$ and ${ }^{87} \mathrm{Sr} /{ }^{86} \mathrm{Sr}$ for the GM, AR, and It 2 surfaces fall outside and below the range defined by the mixing lines, suggesting that their chemistry has been influenced by input from precipitation, or low- ${ }^{87} \mathrm{Sr} /{ }^{86} \mathrm{Sr}$ loess deposition, or both. Data from the It 3 , It 1 , and Sag2 surfaces overlap, consistent with the overlapping ${ }^{87} \mathrm{Sr} /{ }^{86} \mathrm{Sr}$ values for It1 and Sag2 soils in Figure 3, but despite the clear differences in the stream water $\mathrm{Ca} / \mathrm{Sr}$ vs. ${ }^{87} \mathrm{Sr} /{ }^{86} \mathrm{Sr}$ for these surfaces (Fig. 4). This may be because, as discussed above, the cold acid digestible fraction represents an estimate of potentially "easily weatherable" minerals, but does not necessarily reflect what is actually being weathered. Therefore, the It 3 , It1, and Sag2 surfaces could have similar reservoirs of "easily weatherable" minerals as estimated by the digest, yet release into streams very different weathering products that represent the most soluble part of the "easily weatherable" fraction. Cryoturbation may also play a role in keeping the cold acid digestible fraction similar in soils close in age (like the It1 and Sag2) via the upward mixing of lessweathered minerals from deeper, less frequently thawed layers.

Despite the overlapping values of data from the It3, It1, and Sag2 surfaces, samples from the youngest (It2) of the four surfaces with similar parent material generally have the highest $\mathrm{Ca} / \mathrm{Sr}$ and lowest ${ }^{87} \mathrm{Sr} /{ }^{86} \mathrm{Sr}$, while samples from the oldest (Sag1) of these four surfaces have the lowest $\mathrm{Ca} / \mathrm{Sr}$ and highest ${ }^{87} \mathrm{Sr} /{ }^{86} \mathrm{Sr}$, plotting near the Dke digest end-member. These data suggest that carbonate weathering causes detectable geochemical differences to develop in the cold acid digestible fraction of shallow mineral soil over hundreds of thousands of years. Additional research will be necessary to explore the relationship between the time scale at which the effects of weathering are apparent and the rate of cryoturbation.

These results show that carbonate content decreases in soils and carbonate weathering products decrease in streams with increasing surface age, but the carbonate weathering regime is still generally dominant even on older surfaces. This interpretation is consistent with previous studies of aquatic and soil chemistry in this region (Kling et al., 1992) and similar arctic regions (Stutter and Billet, 2003). The decrease in carbonate weathering over time (driven by a depletion of carbonate minerals via weathering) that 


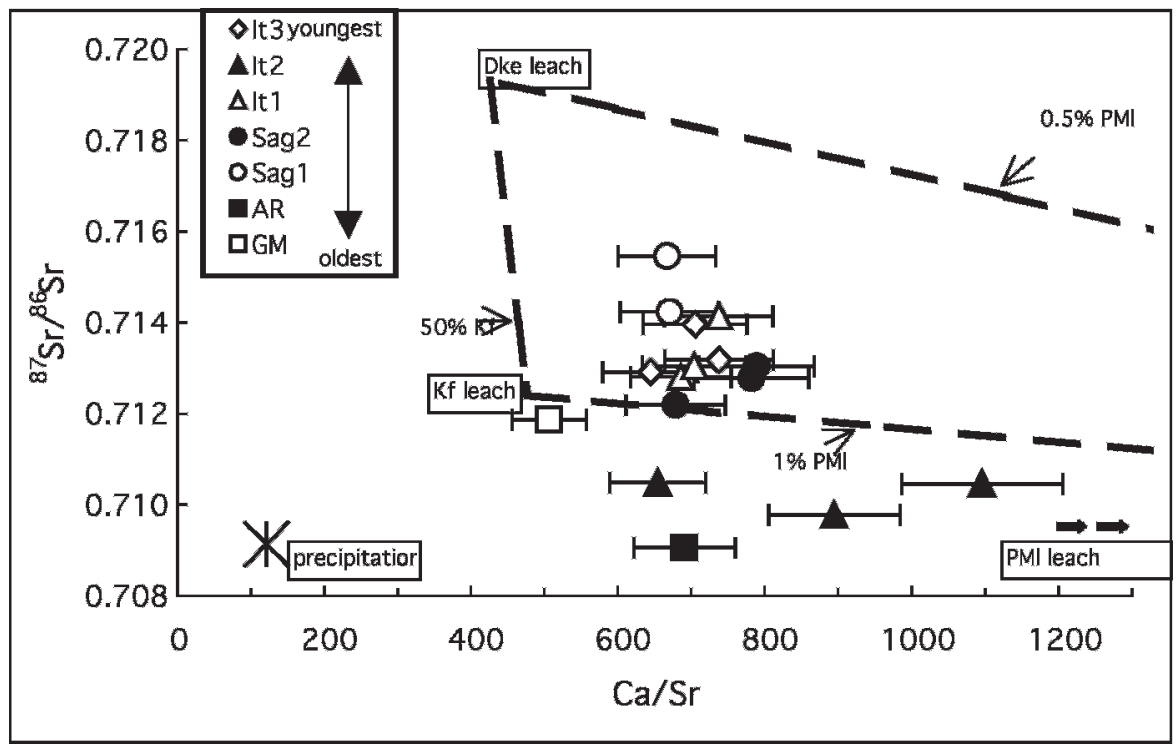

FIGURE 5. Cold acid digestible fraction ${ }^{87} \mathrm{Sr} /{ }^{86} \mathrm{Sr}$ vs. Ca/Sr of soil "A" horizon samples from glacial deposit surfaces. Dashed lines connecting the cold acid digestible fraction values of $\mathrm{PMI}$, Dke, and Kf rock units (see Table 1) represent simple chemical mixing lines for these endmembers, as in Figure 4. $\mathrm{Ca} / \mathrm{Sr}$ is a molar ratio. Error bars represent propagated $\mathrm{Ca} / \mathrm{Sr}$ analytical errors; ${ }^{87} \mathrm{Sr} /{ }^{86} \mathrm{Sr}$ errors are smaller than the symbol size and are reported in Table 3. Rock unit abbreviations are explained in the text.

we interpret from these data is similar to changes that take place in a much shorter time (thousands rather than hundreds of thousands of years) in tropical and temperate climates (e.g. Lichter, 1998). Despite the extended timeframe, this change in the carbonate content of soils with time supports the suggestion of Munroe and Bockheim (2001) that the traditional soil chronosequence model relating age to soil properties is applicable even in cryoturbated soils.

\section{SILICATE WEATHERING}

The presence of dissolved $\mathrm{Si}$ in low but detectable levels in streams on almost every geomorphic surface in our study area suggests that some silicate weathering is occurring; the range of dissolved streamwater Si concentrations in this study is similar to those reported for streams in similar arctic and subarctic systems with sedimentary or mixed bedrock influences (Huh et al., 1998; Millot et al., 2003; Stutter and Billet, 2003; Humborg et al., 2004). The inference that silicate minerals are weathering is consistent with the observed lower concentrations of $\mathrm{K}$ in the cold acid digestible fraction of active-layer mineral soil compared to permafrost on six out of the seven surfaces examined in this study. At least some of this $\mathrm{K}$ is likely derived from the most soluble silicate minerals contained in parent material sandstones, which have from 2 to 10 times the amount of cold acid digestible fraction $\mathrm{K}$ than the limestone unit (Table 1).

\section{NUTRIENT RELEASE VIA MINERAL WEATHERING}

The ecological influence of weathering in this region is reflected in the exchangeable fraction of soils. Exchangeable ${ }^{87} \mathrm{Sr} /{ }^{86} \mathrm{Sr}$ and cold acid digestible fraction ${ }^{87} \mathrm{Sr} /{ }^{86} \mathrm{Sr}$ for each soil horizon are well correlated $\left(R^{2}=0.74\right)$ across all surfaces in this study. This suggests that locally derived mineral weathering products are the primary source of exchangeable $\mathrm{Sr}$, and therefore probably also the source of ecologically important elements like $\mathrm{Ca}, \mathrm{P}$, and $\mathrm{K}$ to the watersheds on these surfaces.

We did not perform soil extractions specifically designed to determine the reservoirs of $\mathrm{P}$ in these soils. However, the ratio of $\mathrm{Ca}$ to $\mathrm{P}$ in the cold acid digestible fraction of active-layer mineral soil samples on all surfaces is consistently greater than or equal to 1.67 (Fig. 6), which is the $\mathrm{Ca} / \mathrm{P}$ of apatite $\left[\mathrm{Ca}_{5}\left(\mathrm{PO}_{4}\right)_{3}(\mathrm{~F}, \mathrm{Cl}, \mathrm{OH})\right]$, the major $\mathrm{P}$-bearing mineral in rocks. $\mathrm{Ca} / \mathrm{P}$ is lowest and nearest to
1.67 in Sag1 soils, which have the least carbonate content as determined by $\mathrm{Ca} / \mathrm{Sr}$ and ${ }^{87} \mathrm{Sr} /{ }^{86} \mathrm{Sr}$. Only 3 out of 52 active-layer mineral soil samples with measurable cold acid digestible fraction $\mathrm{P}$ have a $\mathrm{Ca} / \mathrm{P}$ ratio less than 1.67 (lower by at most only $26 \%$ ), suggesting that apatite is the primary source of $\mathrm{P}$ in this fraction. High $\mathrm{Fe}$ concentrations in the hot acid digestible fraction suggest the possibility that some of the $\mathrm{P}$ in this fraction is bound to $\mathrm{Fe}$ hydroxide species. However, $\mathrm{P}$ and Fe concentrations in this fraction are poorly correlated, so it is likely that $\mathrm{P}$ in this fraction is bound in other (e.g. organic) forms as well.

\section{GEOCHEMICAL IMPLICATIONS OF INCREASING THAW DEPTH}

Our results suggest several possible geochemical consequences of increased active-layer thickness brought about by warming climate. The most conspicuous is increasing carbonate and $\mathrm{Ca}$ supply to soil and stream water with increasing active-layer thickness. This change would be ubiquitous across the study area,

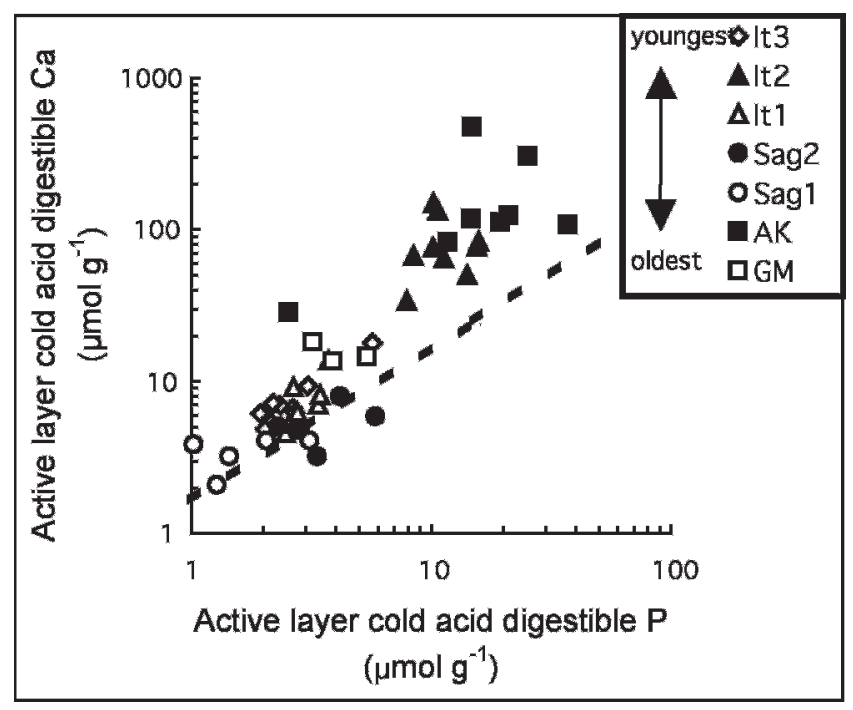

FIGURE 6. Cold acid digestible fraction $\mathrm{Ca}\left(\mu \mathrm{mol} \mathrm{g}^{-1}\right)$ vs. cold acid digestible fraction $\mathbf{P}\left(\mu \mathrm{mol} \mathbf{g}^{-1}\right)$ in active-layer mineral soil samples from different glacial surfaces. The dashed line represents the $\mathrm{Ca} / \mathrm{P}$ of apatite (1.67). Analytical errors are reported in the text. 
as all profiles exhibit geochemical indicators of increasing calcite content with depth, and analysis of data from all surfaces shows that cold acid digestible fraction $\mathrm{Ca}$ concentrations are significantly higher in the permafrost than in the active layer. The increased carbonate in soils brought about by calcite dissolution may lead to increased $\mathrm{pH}$ of acidic tundra, and potentially related changes in vegetation (Bockheim et al., 1998), but these changes would happen concurrently with and could be mitigated by changes in organic matter decomposition and organic acid production that may accompany warmer conditions and deeper thaw.

In addition to increasing carbonate dissolution and general base cation release, our measurements suggest that increased active-layer thickness may increase mineral weathering release of two important nutrients, $\mathrm{P}$ and $\mathrm{K}$. On all surfaces there were significantly greater concentrations of exchangeable $\mathrm{K}$ in the permafrost than in the active-layer mineral soil. Both exchangeable and cold acid digestible fraction $\mathrm{P}$ concentrations were significantly greater in the permafrost than in the active-layer mineral soil overall. The mean increase in $\mathrm{P}$ between these layers was $28 \%$ for the exchangeable fraction and $61 \%$ for the cold acid digestible fraction. The deep permafrost sample from the Sag1 surface was enriched in cold acid digestible fraction $\mathrm{P}$ compared to active-layer soil (including "O" horizons) or shallow permafrost on any surface. Despite these mean differences in $\mathrm{K}$ and $\mathrm{P}$ between permafrost and active-layer mineral soil, the surface-specific differences between active-layer mineral soil and permafrost concentrations of these elements are variable. For example, the It2 surface has active-layer mineral soil concentrations of cold acid digestible fraction $\mathrm{P}$ that are actually higher than shallow permafrost concentrations, whereas the It 3 and Sag1 surfaces have shallow permafrost concentrations of cold acid digestible fraction $\mathrm{P}$ that are more than double the concentrations found in active-layer mineral soil.

These data support the predictions of Hobbie et al. (1999) that thawing of permafrost could increase $\mathrm{P}$ availability to ecosystems and provide more information regarding possible mechanisms. Because the permafrost contains small but significantly greater concentrations of exchangeable $\mathrm{P}$ than the active layer, increases in available $\mathrm{P}$ may occur concurrently with permafrost thawing. Subsequent to initial thaw depth increases, a larger increase in newly available $\mathrm{P}$ may occur gradually as previously frozen minerals weather. Based on the differences in exchangeable and cold acid digestible $\mathrm{P}$ concentration profiles between surfaces, it is reasonable to expect that different geographic areas or watersheds may undergo different degrees of increased $\mathrm{P}$ availability. Some areas, such as those with already high cold acid digestible fraction $\mathrm{P}$ in the active layer (It2 and AR) or those where past weathering of currently frozen soil has depleted $\mathbf{P}$ in the first few centimeters of permafrost (Sag2), may change little in $\mathrm{P}$ availability until large increases (tens of centimeters) in thaw depth occur. Thaw-depth driven increases in $\mathrm{K}$ availability are also likely to be spatially variable, based on differences in the exchangeable and cold acid digestible fraction $\mathrm{K}$ depth profiles among surfaces.

Sulfur concentrations in soil show no statistically significant increase from the active-layer mineral soil to the permafrost across all surfaces for any fraction, but several individual surfaces have higher $\mathrm{S}$ concentrations in the permafrost than in the active-layer mineral soil in either the exchangeable (It3, It2, GM), cold acid digestible (It3, It1), or hot acid digestible (It3, It2, It1) fractions (Tables 2, 3, and 4). The Sag1 deep permafrost sample has exchangeable and cold acid digestible fraction $\mathrm{S}$ concentrations $100 \%$ and $25 \%$ greater, respectively, than any active-layer sample from any surface, including $\mathrm{O}$ horizon samples. Our analyses do not distinguish whether the $\mathrm{S}$ in these soils is inorganically or organically bound, but both forms are likely because the soils are organic rich and the sandstone units of the parent material contain accessory sulfide minerals. Sulfur may be an important control on $\mathrm{Hg}$ methylation via sulfate-reducing bacteria (Compeau and Bartha, 1985; Gilmour et al., 1992); methylation of $\mathrm{Hg}$ in high arctic wetlands is an important step in $\mathrm{Hg}$ bioaccumulation in arctic food chains, although soils there appear to have low levels of sulfatereducing bacteria (Loseto et al., 2004). Further study of the forms and concentrations of $\mathrm{S}$ in the permafrost may shed light on how increased thaw depth will influence $\mathrm{SO}_{4}$ concentrations in arctic surface waters, potentially affecting $\mathrm{Hg}$ methylation pathways.

\section{USE OF STREAM GEOCHEMISTRY AS A MONITOR OF THAW DEPTH}

The geochemical profiles of soils on the central North Slope described in this study may provide a useful tool for qualitatively detecting watershed-scale changes in thaw depth. We hypothesize that in watersheds which have sufficient changes in soil geochemistry with depth, such as the ${ }^{87} \mathrm{Sr} /{ }^{86} \mathrm{Sr}$ or $\mathrm{Ca} / \mathrm{Sr}$ trends described in this study (Fig. 3), stream geochemistry may reflect increases in thaw depth. Specifically, we predict that as thaw depth increases in this study area, more carbonate weathering products will be dissolved in late summer streamwater and will be detectable as increases in streamwater elemental ratios such as $\mathrm{Ca} / \mathrm{Sr}$ and decreases in streamwater ${ }^{87} \mathrm{Sr} /{ }^{86} \mathrm{Sr}$ over periods of many years. On some surfaces with very pronounced geochemical differences between active-layer mineral soil and permafrost, such as the It1 surface, regular analysis of stream geochemistry may signal watershed-scale permafrost degradation before it is detectable by traditional physical (e.g., steel probe) methods of measuring thaw depth. Even in permafrost regions with non-carbonate-dominated soil parent material, less intensely weathered soil at depth may provide a geochemical signature as soils thaw more deeply.

\section{Conclusions}

Analysis of stream and soil geochemistry on arctic Alaskan glacial till deposits of varying ages indicates that carbonate weathering is the major controlling factor of geochemical trends related to soil depth and surface age. Carbonate minerals have been depleted by weathering in the active layer on every geomorphic surface, and on the four surfaces with similar parent material, stream and soil geochemistry suggests progressive carbonate depletion with time over hundreds of thousands of years. Dissolved Si concentrations in streams are similar to those in other arctic watersheds, suggesting that silicate weathering is also occurring on these surfaces, but at a much slower rate than carbonate weathering.

Compared to active-layer mineral soils, permafrost has overall greater exchangeable $\mathrm{K}$ concentrations, exchangeable and acid digestible $\mathrm{P}$ and $\mathrm{Ca}$ concentrations, and carbonate content. Some glacial deposit surfaces also have higher $\mathrm{S}$ concentrations in permafrost than in active-layer soils. The magnitude of the geochemical differences between permafrost and active-layer soils varies among different surfaces. The results of this study suggest that increasing thaw depth brought about by climate change will lead to overall (albeit spatially variable) increases in carbonate, $\mathrm{Ca}, \mathrm{P}, \mathrm{K}$, and $\mathrm{S}$ supply to soils and streams. This increase may occur concurrently with increased maximum thaw as the exchangeable fraction becomes available, and then progressively as soluble minerals begin to weather. The nature of these 
geochemical changes will depend on the rate of thaw; changes are likely to be gradual overall but could be locally abrupt in areas of rapid thermokarst formation. Based on geochemical differences between active-layer soil and permafrost, stream geochemistry may be a useful qualitative monitor of watershed-scale changes in thaw depth.

\section{Acknowledgments}

We thank D. Zak, H. Adams, and K. Judd for help in the field. Funds for field research were provided by grants from the Geological Society of America and the University of Michigan Geological Sciences and Ecology and Evolutionary Biology Departments. Research was also supported by NSF DEB0423385, DEB 97-26837, ATM-0439620, and ARC-0435893. We appreciate thoughtful reviews by Michael Gooseff, Suzanne Anderson, and two anonymous reviewers.

\section{References Cited}

Anisimov, O. A., Shiklomanov, N. I., and Nelson, F. E., 1997: Global warming and active-layer thickness: results from transient general circulation models. Global and Planetary Change, 15: 61-77.

Blum, J. D., Klaue, A., Nezat, C. A., Driscoll, C. T., Johnson, C. E., Siccama, T. G., Eagar, C., Fahey, T. J., and Likens, G. E., 2002: Mycorrhizal weathering of apatite as an important calcium source in base-poor forest ecosystems. Nature, 417: 729-731.

Bockheim, J. G., Walker, D. A., Everett, L. R., Nelson, F. E., and Shiklomanov, N. I., 1998: Soils and cryoturbation in moist nonacidic and acidic tundra in the Kuparuk River Basin, Arctic Alaska, U.S.A. Arctic and Alpine Research, 30: 166-174.

Brown, J., and Krieg, R. A., 1983: Guidebook to permafrost and related features along the Elliot and Dalton Highways, Fox to Prudhoe Bay, Alaska. Fairbanks: University of Alaska-Fairbanks.

Brown, J. G., Kling, G. W., Hinkel, K. M., Hinzman, L. D., Nelson, F. E., Romanovsky, V., and Shiklomanov, N. I., 2002: Arctic Alaska and Seward Peninsula. In Brown, J. G., Hinkel, K. M., and Nelson, F. E. (eds.), The circumpolar active layer monitoring (CALM) program: research designs and initial results. Polar Geography, 24: 165-258.

Compeau, G. C., and Bartha, R., 1985: Sulfate-reducing bacteria: principal methylators of mercury in anoxic estuarine sediment. Applied Environmental Microbiology, 50: 498-502.

Everett, K. R., Marion, G. M., and Kane, D. L., 1989: Seasonal geochemistry of an arctic tundra drainage-basin. Holarctic Ecology, 12: 279-289.

Giblin, A. E., Nadelhoffer, K. J., Shaver, G. R., Laundre, J. A., and McKerrow, A. J., 1991: Biogeochemical diversity along a riverside toposequence in arctic Alaska. Ecological Monographs, 61: 415-436.

Gilmour, C. C., Henry, E. A., and Mitchell, R., 1992: Sulfate stimulation of mercury methylation in fresh-water sediments. Environmental Science \& Technology, 26: 2281-2287.

Hamilton, T. D., 1978: Surficial geologic map of the Philip Smith Mountains quadrangle, Alaska. Reston, VA: U.S. Geological Survey, Map MF-879.

Hamilton, T. D., 1986: Late Cenozoic glaciation of the Central Brooks Range. In Hamilton, T. D., Reed, K. M., and Thorson, R. M. (eds.), Glaciation in Alaska: the geologic record. Anchorage, AK: Alaska Geological Society, 265 pp.

Hamilton, T. D., 1994: Late Cenozoic glaciation of Alaska. In Plafker, G., and Berg, H. C. (eds.), The geology of Alaska. Boulder, CO: Geological Society of America, The Geology of North America, vol. G-1.
Hamilton, T. D., 2003: Glacial geology of Toolik Lake and the Upper Kuparuk River region. Fairbanks, AK: Alaska Geobotany Center, Institute of Arctic Biology, University of AlaskaFairbanks, 24 pp.

Hamilton, T. D., and Porter, S. C., 1975: Itkillik glaciation in the Brooks Range, northern Alaska. Quaternary Research, 5: 471-497.

Haugen, R. K., 1982: Climate of remote areas in north-central Alaska, 1975-1979 summary. Hanover, NH: U. S. Army Cold Regions Research and Engineering Laboratory, Report 110.

Heiri, O., Lotter, A. F., and Lemcke, G., 2001: Loss on ignition as a method for estimating organic and carbonate content in sediments: reproducibility and comparability of results. Journal of Paleolimnology, 25: 101-110.

Hobbie, J. E., Peterson, B. J., Bettez, N., Deegan, L., O'Brien, W. J., Kling, G. W., Kipphut, G. W., Bowden, W. B., and Hershey, A. E., 1999: Impact of global change on the biogeochemistry and ecology of an Arctic freshwater system. Polar Research, 18: 207-214.

Hobbie, S. E., Miley, T. A., and Weiss, M. S., 2002: Carbon and nitrogen cycling in soils from acidic and nonacidic tundra with different glacial histories in Northern Alaska. Ecosystems, 5: $761-774$

Huh, Y., Tsoi, M.-Y., Zaitsev, A., and Edmond, J. M., 1998: The fluvial geochemistry of the rivers of Eastern Siberia: I. Tributaries of the Lena River draining the sedimentary platform of the Siberian Craton. Geochimica et Cosmochimica Acta, 62: 1657-1676.

Humborg, C., Smedberg, E., Blomqvist, S., Morth, C.-M., Brink, J., Rahm, L., Danielsson, A., and Sahlberg, J., 2004: Nutrient variations in boreal and subarctic Swedish rivers: landscape control of land-sea fluxes. Limnology and Oceanography, 49: 1871-1883.

Judd, K. E., and Kling, G. W., 2002: Production and export of dissolved $\mathrm{C}$ in arctic tundra mesocosms: the roles of vegetation and water flow. Biogeochemistry, 60: 213-234.

Kling, G. W., O’Brien, J., Miller, M. C., and Hershey, A. E., 1992: The biogeochemistry and zoogeography of lakes and rivers in arctic Alaska. Hydrobiologia, 240: 1-14.

Lichter, J., 1998: Rates of weathering and chemical depletion in soils across a chronosequence of Lake Michigan sand dunes. Geoderma, 85: 255-282.

Loseto, L. L., Siciliano, S. D., and Lean, D. R. S., 2004: Methylmercury production in High Arctic wetlands. Environmental Toxicology and Chemistry, 23: 17-23.

Menzie, W. D., Reiser, H. N., Brosge, W. P., and Detterman, R. L., 1985: Map showing distribution of mineral resources (excepting oil and gas) in the Philip Smith Mountains quadrangle, Alaska. Reston, VA: U.S. Geological Survey, Map MF-879-C.

Millot, R., Gaillardet, J., Dupre, B., and Allegre, C. J., 2003: Northern latitude chemical weathering rates: clues from the Mackenzie River Basin, Canada. Geochimica et Cosmochimica Acta, 67: 1305-1329.

Moore, T. E., Wallace, W. K., Bird, K. J., Karl, S. M., Mull, C. G., and Dillon, J. T., 1994: Geology of northern Alaska. In Plafker, G., and Berg, H. C. (eds.), The Geology of Alaska. Boulder, CO: Geological Society of America, The Geology of North America, vol. G-1.

Mull, C. G., and Adams, K. E. (eds.), 1985: Bedrock Geology of the eastern Koyukuk Basin, central Brooks Range, and east central Arctic Slope along the Dalton Highway, Yukon River to Prudhoe Bay. Anchorage, AK: Alaska Department of Natural Resources, Division of Geological and Geophysical Surveys, Guidebook 7:309 pp.

Munroe, J. S., and Bockheim, J. G., 2001: Soil development in low-arctic tundra of the northern Brooks Range, Alaska, USA. Arctic and Alpine Research, 33: 78-87.

Nadelhoffer, K. J., Giblin, A. E., Shaver, G. R., and Laundre, J. A., 1991: Effects of temperature and substrate quality on element mineralization in six arctic soils. Ecology, 72: 242-253. 
Palmer, M. R., and Edmond, J. M., 1992: Controls over the strontium isotope composition of river water. Geochimica et Cosmochimica Acta, 56: 2099-2111.

Ping, C. L., Bockheim, J. G., Kimble, J. M., Michaelson, G. J., and Walker, D. A., 1998: Characteristics of cryogenic soils along a latitudinal transect in Arctic Alaska. Journal of Geophysical Research-Atmospheres, 103: 28917-28928.

Schlesinger, W. H., 1997: Biogeochemistry: an analysis of global change. Second edition. San Diego: Academic Press.

Shaver, G. R., and Chapin, F. S., 1995: Long-term responses to factorial NPK fertilizer treatment by Alaskan wet and moist tundra sedge species. Ecography, 18: 259-275.
Stutter, M. I., and Billet, M. F., 2003: Biogeochemical controls on streamwater and soil solution chemistry in a High Arctic environment. Geoderma, 113: 127-146.

Tedrow, J. C. F., Drew, J. V., Hill, D. E., and Douglas, L. A., 1958: Major genetic soils of the arctic slope of Alaska. Journal of Soil Science, 9: 33-45.

Walker, J. C. G., Hays, P. B., and Kasting, J. F., 1981: A negative feedback mechanism for the long-term stabilization of Earth's surface temperature. Journal of Geophysical Research, 86: 9776-9782.

Ms accepted February 2006 